\title{
High precision particle astrophysics as a new window on the universe with an Antimatter Large Acceptance Detector In Orbit (ALADInO)
}

\section{R. Battiston, et al. [full author details at the end of the article]}

Received: 4 August 2020 / Accepted: 9 February 2021 / Published online: 19 May 2021

(C) The Author(s) 2021, corrected publication 2021

\begin{abstract}
Multimessenger astrophysics is based on the detection, with the highest possible accuracy, of the cosmic radiation. During the last 20 years, the advent space-borne magnetic spectrometers in space (AMS-01, Pamela, AMS-02), able to measure the charged cosmic radiation separating matter from antimatter, and to provide accurate measurement of the rarest components of Cosmic Rays (CRs) to the highest possible energies, have become possible, together with the ultra-precise measurement of ordinary CRs. These developments started the era of precision Cosmic Ray physics providing access to a rich program of high-energy astrophysics addressing fundamental questions like matter-antimatter asymmetry, indirect detection for Dark Matter and the detailed study of origin, acceleration and propagation of CRs and their interactions with the interstellar medium.

In this paper we address the above-mentioned scientific questions, in the context of a second generation, large acceptance, superconducting magnetic spectrometer proposed as mission in the context of the European Space Agency's Voyage2050 long-term plan: the Antimatter Large Acceptance Detector In Orbit (ALADInO) would extend by about two orders of magnitude in energy and flux sensitivity the separation between charged particles/anti-particles, making it uniquely suited for addressing and potentially solving some of the most puzzling issues of modern cosmology.
\end{abstract}

Keywords Cosmic ray · Antimatter · Dark matter · Magnetic spectrometer

\section{Introduction}

The Science Theme that we propose in this White Paper - High Precision Particle Astrophysics - addresses fundamental topics across particle physics, astrophysics and cosmology through the accurate measurement of Cosmic Rays in space. The theme poses three fundamental questions: 1) What is the origin of the Matter-Antimatter asymmetry in our Universe? 2) What is the particle nature of Dark Matter and does it contribute to the cosmic radiation? 3) Which is the origin, the acceleration and the propagation mechanism of Cosmic Rays? During the last 25 years, these questions have been addressed with increasing accuracy and experimental performances by a 
number space experiments like AMS-01, PAMELA, AGILE, FERMI, AMS-02, CALET, and DAMPE. The improved experimental accuracy has been accompanied by a wealth of results on topics from fundamental properties of particle and fields, to astrophysics, to cosmology. At present, this scientific field has reached a solid maturity and it is exhibiting a very interesting discovery potential complementary and competitive with ground-based experiments like the LHC at CERN or underground experiments like those performed at the Gran Sasso Laboratory. Antimatter particles in CRs represent a small fraction of the total flux, about $5 \times 10^{-3}$ for $\mathrm{e}^{+}, 10^{-5}$ for anti-p, $10^{-7}$ for anti-D, less than $10^{-8}$ for anti- ${ }^{4} \mathrm{He}$ or heavier antinuclei: these tiny fluxes, however, carry a great amount of information, since the origin of antiparticles is intimately related to fundamental processes.

High statistics, accurate measurement of the antimatter component of CRs to the highest possible energies, together with the ultra-precise measurement of ordinary CRs, would provide a rich program of high-energy astrophysics as has been demonstrated by the amazing results of AMS-01, PAMELA, and AMS-02, the first generation of particle spectrometers in space.

Matter-antimatter asymmetry is one of the greatest mysteries of current cosmology: we have no evidence so far from ground experiments for a mechanism which could explain baryogenesis. Anti-D, anti- ${ }^{3} \mathrm{He}$, and anti- ${ }^{4} \mathrm{He}$ have been produced with the same relative rates as $\mathrm{D}$ and ${ }^{3,4} \mathrm{He}$ in heavy ions collisions. Anti-H atoms exhibit exactly the same emission spectra as $\mathrm{H}$ atoms. Limits on proton lifetime does not provide evidence for baryon number violating interactions. At the same time, theories that either predict the existence of nuclear antimatter in segregated domains or its total absence in the current Universe have no firm foundation in observational data. The detection of anti- ${ }^{4} \mathrm{He}$ or heavier antinuclei in CRs would shatter our current understanding of early Universe physics with broad cosmological implications or reveal something completely unforeseen in the realm of astrophysics, such as e.g. an anomalous abundance of dense antibaryon stars or anti-cloudlets in our Galaxy.

The existence of Dark Matter (DM) is well established and supported by a variety of astrophysical and cosmological observations. Its particle nature is still the subject of exciting speculations since no evidence has been found, neither in direct production at the LHC, nor in direct detection at underground experiments, over an amazingly large interval of masses and physical properties. Since DM is abundant in the Universe, particle astrophysics in space is the right environment for indirect searches of DM particles. Antiparticles $\left(\mathrm{e}^{+}, \mathrm{p}^{-}, \mathrm{D}^{-}\right.$, anti- $\left.{ }^{3} \mathrm{He}\right)$ constitute a golden channel for this search, since the rare antiparticle component of DM annihilation/decay products, would give rise to an observable excess on these fluxes with respect to the tiny standard astrophysical expectations, not influenced by the otherwise large background of standard CRs. The knowledge of the astrophysical antiparticle background, would require, however, a substantial improvement to match the new sensitivity regime we are aiming for.

This adds a further, essential topic to our Science Theme, namely the origin, acceleration, and propagation of CRs and their interactions with the interstellar medium. This topic addresses a rich program of measurements of the cosmic radiation: energy spectra, chemical and isotopic composition, arrival directions, and temporal variations at still unexplored sensitivities and energies, reaching, for the lighter CR 
components ( $\mathrm{p}, \mathrm{He})$ up to $\mathrm{PeV}$ energies, the so-called "knee" region, where a flux transition from galactic to extragalactic CR sources is expected.

In order to address the above-mentioned scientific questions, we also discuss a set of key requirements for a mission based on a large acceptance, superconducting magnetic spectrometer to separate charged particles/anti-particles (Antimatter Large Acceptance Detector In Orbit -ALADInO).

Access to space is mandatory to avoid atmospheric background and to ensure an extended observation time. A collection factor of $\approx 20 \mathrm{~m} 2$-sr years is needed to increase by a factor $10^{3}$ current limits on anti- ${ }^{4} \mathrm{He}$ or heavier nuclear anti-matter. A collection factor of $\sim 15 \mathrm{~m}^{2}$ sr years, a maximum detectable rigidity of $20 \mathrm{TV}$ and $\mathrm{e}^{ \pm} / \mathrm{p}^{ \pm}$separation at the level of $10^{6}$ are needed to provide a substantial progress in the field. The same collection factor, together with a velocity measurement with $1 \%$ resolution, would allow for the measurement of $\mathrm{O}(1 \mathrm{GeV})$ anti-p, anti-D and anti- ${ }^{3} \mathrm{He}$, in an energy range where DM models predict signals 1-2 orders of magnitude larger than the astrophysical background. With the above collection factor, the study of the lighter CR spectrum could also reach the energy range $\mathrm{E} \sim 1 \mathrm{PeV}$ shedding light on the puzzle of the composition of extragalactic cosmic rays.

All these requirements are summarized in the concept of ALADInO presented at the end of this paper. ALADInO is based on a High Temperature Superconducting (HTS) magnet, and designed for a five-year mission either on L2 or on High Earth Orbit, in order to maximize the CR collection while minimizing active cooling of the cryomagnet. The key technological challenge in ALADInO is the development of the superconducting toroid making use of HTS and operating at $\mathrm{T} \approx 15-30 \mathrm{~K}$. R\&D activities for the use of HTS in space were already started under the EU-FP7 project SR2S and can further profit in the coming years from the intense effort ongoing at CERN for future experiments at the next generation of high energy colliders.

The ALADInO sub-detectors can be based on well-known technology with high TRL developed for high-energy physics experiments: silicon and sensors, scintillators and homogeneous calorimeters have been successfully operated in long-term missions (AGILE, AMS-01/02, Fermi, PAMELA, CALET, DAMPE) or proposed for future ones (ExTP, CSES2, HERD). For the Voyage 2035-2050 program a new generation of detectors/electronics will be available and tested (e.g. SiPM, silicon pixel detectors) allowing to reach even better performances with reduced power requirements.

In this proposal ALADInO is discussed at the level of concept, which can be implemented also through intermediate phases in order to test the more demanding technologies while addressing part of the physics program.

\subsection{Science case}

Our planet is constantly bombarded by Cosmic Rays (CRs), high-energy charged particles reaching us from regions of space external to the Solar System. The origin of CRs remains a century-old puzzle also considering the fact that, before reaching Earth, their directions are randomized by the interstellar magnetic fields. The leading paradigm is that the bulk of CRs are generated in Galactic processes such as supernova explosions or stellar winds, although extragalactic sources and secondary production by primary CRs with the Interstellar Medium (ISM) may contribute as well to the observed flux. These particles are thus a genuine sample of solar, galactic, and extragalactic 
matter that includes all known nuclei and their long-lived isotopes, as well as electrons and antiparticles. This makes the $\mathrm{CR}$ radiation a unique messenger to probe highenergy phenomena. The CR energy spectrum extends several orders of magnitude from $\sim 10^{6} \mathrm{eV}$ to about $\sim 10^{21} \mathrm{eV}$. Since their discovery at the beginning of last century, scientists have been intrigued by the physical mechanisms of their origin, acceleration and propagation. CRs are a unique kind of messenger connecting fundamental physics, cosmology, and astrophysics. Notable examples of open issues which can be addressed by the study of CR are the still unexplained asymmetry between matter and antimatter in our Universe and the puzzling nature of Dark Matter, six times more abundant than matter.

It follows that the study of high-energy antiparticles in the cosmic radiation may have groundbreaking consequences for cosmology, astrophysics, and particle physics [1].

Nuclear Antimatter seems to be extremely rare in today's visible Universe: most of the antiparticles produced after the post-inflation reheating seems to have disappeared during the baryonic annihilation phase, although convincing mechanisms for this asymmetry have not been put forward. The resulting matter-antimatter asymmetry is one of the greatest puzzles in contemporary cosmology and astrophysics. At extragalactic scales, large concentrations of nuclear antimatter could still exist, but no evidence has been found yet [2-4]. Unambiguous evidence for anti- ${ }^{4} \mathrm{He}$ or heavier antinuclei in CR would have profound consequences on particle physics and cosmology.

The nature of the Dark Matter (DM) is another unsolved problem in contemporary astrophysics. This topic has motivated a varied, worldwide experimental effort to search for signatures of new physics via complementary approaches: underground laboratories (DM direct search), particle colliders (DM production) and observatories on ground and in space (indirect DM search). If the dark halo of the Milky Way is constituted by a new kind of weakly interacting elementary particle with a mass of the order of the electroweak scale then the annihilation or decay of these particles could give rise to a detectable signal, in the form of an excess in the cosmic radiation [5]. An anomalous amount of antiparticles ( $e^{+}$, anti-p, anti-D, anti- $\left.{ }^{3} \mathrm{He}\right)$, could be observable as a distortion of the expected antiparticle spectra in CR. Indirect DM search in the cosmic radiation has motivated experiments aiming with increasing sensitivity at this goal. However, a claim of DM signature from indirect measurements would be challenging, since the production of antiparticles can be due to other mechanisms e.g. in the collisions of ordinary CRs with the interstellar matter. The level of antiparticles arising from these collisions has to be carefully calculated and it constitutes the astrophysical background for indirect DM search [6, 7]. While calculations are reaching an increasing level of complexity and reliability $[8,9]$, the existing models of astrophysical backgrounds crucially depend on our knowledge of CR acceleration and propagation processes through the Galaxy, which are still poorly understood. Galactic sources of CRs, such as supernova remnants, should be able to accelerate CR particles via diffusive shock acceleration mechanisms up to the $\mathrm{PeV}$ energy scale $\left(\sim 10^{15} \mathrm{eV}\right)$ [10]. Their subsequent propagation is governed by diffusive transport in interstellar magnetic turbulence, which keeps CRs confined within the halo of the Milky Way for $\sim 0.1-10$ million years. During this propagation, CRs are subjected to Coulomb/ionization losses and hadronic interactions with the interstellar gas. Along 
with re-shaping their energy spectrum, interactions of CRs with interstellar matter could produce also secondary $\mathrm{CR}$ particles, that is particles that are not accelerated at the sources. $\mathrm{D}$ or ${ }^{3} \mathrm{He}$ isotopes, $\mathrm{Li}-\mathrm{Be}-\mathrm{B}$ isotopes and, most notably, antiparticles are some of the most interesting secondary CRs.

The physics mechanisms regulating the antiparticle background are the same as those producing the other secondary components. Accurate measurements of CR nuclei can consequently be used to calibrate the astrophysical models and enhance the sensitivity for possible anomalous antiparticle signals. The Boron to Carbon $(\mathrm{B} / \mathrm{C})$ ratio is one of the most important physics observables used to assess the expected antiparticle background [11].

Other important processes affecting the near-Earth CR spectrum are related to heliophysics and space science. Charged particles are affected by solar generated magnetic turbulence and solar wind disturbances, which significantly "modulates" the shape of their energy spectrum. The solar modulation effect is a remarkable time-, space-, energy- and particle-dependent phenomenon, which is known to be connected with the Sun's magnetic activity, although the detailed mechanisms of CR interactions with the heliospheric plasma are still subjected to intense research $[12,13]$.

The remaining part of this section is devoted to a better description of the various science topics addressed by our science theme.

Particle Astrophysics addresses key science questions at the interface between particle physics, astrophysics and cosmology:

What is the origin of the antimatter-matter asymmetry in our Universe?

What is the particle nature of dark matter?

Which are the sources of CRs? How and where are they accelerated?

How do they propagate through the Galaxy? What kind of interactions do they experience?

To what extent do extragalactic particles contribute to the CR flux?

Which are the physics processes that rule CR transport in the heliosphere?

High precision investigation of the energy spectrum, composition, arrival directions, and temporal variations of CR components over an extended energy interval is the key to answer or to better understand these open questions.

Nuclear antimatter The detection of complex forms of antinuclei such as anti- ${ }^{4} \mathrm{He}$ or heavier species would cause a profound impact on our understanding of the early Universe. In fact, antinuclei can be regarded as direct indicators for survival of primordial antimatter and for the possible existence of anti-nucleosynthesis processes at work in the core of anti-stars. Nuclear antimatter production in CR collisions and/or from DM sources is extremely suppressed for nuclear species above anti- ${ }^{3} \mathrm{He}[4,14]$. Efforts to search for nuclear antimatter in CR have been developed during the last 15 years $[15,16$, 17]: no observation of anti- ${ }^{4} \mathrm{He}$ or heavier antinuclei in the CR flux has been published down to the level of $10^{-7}$. The lack of unambiguous annihilation signatures from $\gamma$-ray sky surveys led various authors to conclude that the distance to any hypothetical domain dominated by primordial antimatter must be comparable to the horizon scale [18].

The basic recipe to explain the evolution to a baryon asymmetric Universe from a symmetric one has been introduced by Sakharov [19]. It involves a mechanism of baryosynthesis requiring violation of quantum numbers for which there is not any experimental evidence: neither baryon non-conservation nor large levels of CP- 
violation have been observed. The detection of anti- ${ }^{4} \mathrm{He}$ or heavier nuclei would point to the existence of primordial antimatter from the Big Bang nucleosynthesis, while heavier particles such as ${ }^{12} \overline{\mathrm{C}}$ would point to the existence of stellar nucleosynthesis, or dense lumps of antimatter at galactic scale [20, 3, 4].

Very recently, tantalizing hints of anti- ${ }^{3} \mathrm{He}$ and anti- ${ }^{4} \mathrm{He}$ candidates have been presented by the AMS-02 magnetic spectrometer [21]. Considering the huge helium statistics, about one antihelium candidate out of 100 million helium events, a daunting simulation effort is required to exclude misidentification of these events. Even if the results will be published and substantiated by a convincing and robust simulation, the enormous relevance of this result would require confirmation and extension by another experiment with different systematic uncertainties and able to collect a much larger statistical sample.

Observation and detailed study of nuclear antimatter would be a game changer in our understanding of the physics of the early Universe and of the fundamental properties of particle and fields.

Dark matter In spite of the lack of evidence for Beyond-Standard-Model (BSM) particles from experiments at the Large Hadron Collider (LHC), TeV-scale models of DM particles are nicely supported by cosmological constraints. The thermal freeze-out mechanism of Weakly Interacting Massive Particles (WIMPs) naturally predicts the required level of abundance of cosmological DM in today's Universe in full accordance with cosmological observations [22, 23]. TeV-scale WIMP-DM candidates have production cross-sections which are extremely suppressed at the LHC [24, 25]; even prospects for future colliders are not encouraging [26]. Their direct detection via elastic scattering off nuclei is also disfavored for the multi-TeV masses [27]. On the other hand, indirect search appears to be the most promising approach for TeV-scale WIMPlike DM candidates. In fact, for these candidates, there is a large region of the parameter space consistent both with Fermi-LAT $\gamma$-ray data and the CMB limits $[5,28-30]$ as can be seen in Fig. 1.
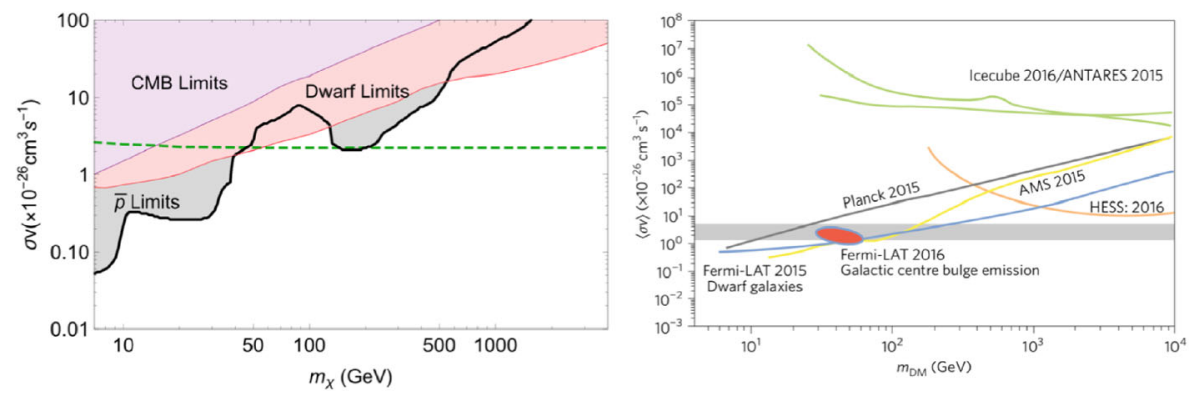

Fig. 1 Left from [31]: upper limits on the DM annihilation rate to b-quark pairs derived from CR antiproton measurements from AMS-02 (black), along with the regions excluded by the Planck observations of the CMB (purple) and by $\gamma$-ray data on dwarf galaxies (red). In can be seen that the regions of DM parameter space favored by AMS-02 lie either in the TeV region of DM mass or in the 50-100 GeV mass region, both consistent with the thermal DM annihilation cross-section level (green dashed line). The $50 \mathrm{GeV}$ DM is also favored by the Fermi-LAT $\gamma$-ray data, i.e. the so-called Galactic Centre excess. Right from [32]: multimessenger constraints on the DM annihilation to b-quark pairs, using neutrinos, CMB photons, $\gamma$-rays, and antiprotons. The best-fit DM interpretation of the Galactic Centre excess is shown as red circle. The thermal DM annihilation cross section is shown as a gray shaded band 
The most promising channels for indirect DM searches are those involving antimatter in the final state, e.g. elementary antiparticles such as $\mathrm{e}^{+}$, anti-p or anti-nuclei such as anti-D, anti- ${ }^{3} \mathrm{He}$. In recent years, this field has witnessed significant advancements on both experimental and theoretical sides, stimulated by new-generation space-borne experiments such as PAMELA, the Fermi-LAT telescope, and the Alpha Magnetic Spectrometer AMS-02. All the experiments observed a very significant and unexpected excess in the positron flux above $10 \mathrm{GeV}$ : the positron energy spectrum is significantly different to the electron one and it is not compatible with the expected astrophysical background. It is now generally accepted that the positron and electron spectra can be jointly understood only assuming the existence of as yet unknown sources of high-energy positrons and electrons in the Galaxy.

These observations were possible only because of the outstanding accuracy of the new generation of space-borne experiments and of the high statistics samples which have been collected and analyzed. The positron excess has caused a huge interest. During the last decade, hundreds of papers have proposed diverse interpretations, either invoking annihilation/decay of DM particles [33-45], nearby astrophysical sources such as pulsars or supernova remnants [46-58] or modifications to the CR acceleration and propagation mechanisms [59-64]. These predictions are all compatible with the accuracy of current data; additional information is needed to disentangle the dominating sources of high energy $\mathrm{e}^{ \pm}$.

In order to better understand the source of this excess, also the arrival directions of high-energy CRs can be studied, searching for anisotropy signals which could be associated with localized sources. High-energy leptons lose rapidly their energy while they travel in the ISM; in order to reach the Earth, those detected at the highest energies must have been produced in the vicinity of the Solar System (within $\sim 1 \mathrm{kpc}$ ). Localized sources of CR positrons may then be detectable as an excess in given directions of the sky, favoring or disfavoring particular models.

Precision measurements of the detailed features of the energy spectra and arrival directions of CR positrons and electrons at the $\mathrm{TeV}$ scale are needed to clarify whether DM annihilations or new astrophysics phenomena are the source of the anomalies observed on the positron flux.

Evidence for detectable DM annihilation signals in the hadronic channels would significantly strengthen the DM hypothesis. No astrophysical sources would produce at the same time a distortion on the positron and antiproton spectra. In this respect, an ideal signature of DM would be the observation of an excess of CR antiprotons at high energies, compatible with the DM candidate mass and decay branching ratios. The scenario of a WIMP-like DM annihilating into hadronic channels (e.g. quark-antiquark pairs) is well consistent with astrophysical $\gamma$-ray bounds and it is well motivated by theories Beyond Standard Model, especially considering the absence of new physics signals from LHC $[25,31,90,65]$. To find out whether a DM signal is hidden in the $\mathrm{CR}$ antiproton flux a reliable assessment of astrophysical background models is needed and depending upon the DM mass, antiproton measurements needs to be extended above the TeV scale.

Light antinuclei, in particular anti-D and anti- ${ }^{3} \mathrm{He}$, represent the most promising channel for DM searches in terms of signal-to-background ratio. In fact, due to kinematics, the astrophysical background of CR anti-D or anti- ${ }^{3} \mathrm{He}$ is highly suppressed in the low energy region associated with WIMP-DM models. The detection 
of anti-D or anti- ${ }^{3} \mathrm{He}$ excess in CRs is often considered as a "smoking gun" for DM searches. On the other hand, a null result would provide strong exclusion limits for a wide range of viable DM models.

Accurate measurements of anti-protons, anti-deuterons, and anti- ${ }^{3} \mathrm{He}$ in $\mathrm{CR}$ are the key to investigate $\mathrm{GeV}$ to $\mathrm{TeV}$ scale DM models.

Multi-channel investigation of rare CR components is central to probe/study DM provided that uncertainties on the astrophysical backgrounds are significantly reduced by means of a precise knowledge of the composition and energetics of cosmic rays in the galaxy and the heliosphere.

CR protons and nuclei Accurate, recent measurements of the most abundant CR species, such as protons and helium have recently revealed new and unexpected features in their energy spectra, that may shed new light on the acceleration and propagation mechanisms of CRs in the Galaxy. The longstanding simple picture of a featureless and universal power-law energy spectrum of CRs might have been finally overcome $[48,66]$. A remarkable change of spectral indices has been established for several CR energy spectra at a kinetic energy of about $300 \mathrm{GeV}$. The change in slope, consisting of a spectral hardening, has been suggested by CREAM, measured by PAMELA for $\mathrm{p}$ and He, and then accurately characterized by AMS-02 for all nuclear species up to Oxygen. Such a spectral hardening appears to be universal, in the sense that it has been detected in all charged nuclear species. However, the strength of the spectral changes appears to be more pronounced for secondary species (such as Li-Be$\mathrm{B}$ nuclei) in comparison to primary elements (such as $\mathrm{p}, \mathrm{He}, \mathrm{C}$, or $\mathrm{O}$ ): this suggests that a revised description of the $\mathbf{C R}$ diffusive propagation is needed. At higher energies, recent calorimetric measurements reported by CREAM-III and CALET hint at the appearance of additional features in the proton energy spectrum, although the situation requires experimental clarification $[67,68]$.

On top of the hardening, the precision of recent data revealed that the rigidity spectrum of CR protons is steeper than the spectrum of helium nuclei, with the puzzling result that $\mathrm{CR}$ helium could become more abundant than hydrogen (proton) at the energy above $\sim 10 \mathrm{TeV}$. Theoretically, the different slope between proton and helium suggests possible mechanisms of violation of universality in CR acceleration, in contrast to the basic prescription of the diffusive-shock-acceleration theory. It is unclear, at present, whether $Z>2$ heavier nuclei will follow the same energy spectrum of helium. Additional features may have been found by recent AMS-02 measurements of N, S, Si [69] and in the spectrum of heavy nuclei such as Iron [70].

The current situation therefore demands an experimental clarification at multi$\mathrm{TeV}$ energy and high- $\mathrm{Z}$ charges which can only be provided by a high-precision spectrometric measurement of the individual nuclear species encompassing the relevant energy regions where indications for spectral features are emerging. Evidence for the observed hardenings will then be checked and better quantified, therefore providing strong constraints to state-of-the-art astrophysical models of $\mathrm{CR}$ acceleration and propagation mechanisms, also studying the possibility of a contribution of nearby sources. In particular, strong constraints are expected from multi-TeV measurements of the energy spectrum of secondary nuclei, along with secondary-to-primary ratios, which will provide a robust baseline of the astrophysical antimatter background. 
Precise measurements of primary and secondary CR nuclei above $\mathrm{TeV}$ are fundamental to understand the acceleration and propagation mechanisms of CRs in the Galaxy and to constrain models of astrophysical backgrounds in indirect DM searches.

Since the first experimental evidence [71], the energy region around the knee in the all-particle cosmic ray spectrum, at about $3 \mathrm{PeV}$, has been investigated by many experiments with different approaches [72]. Several theoretical explanations have been proposed exploiting different hypotheses on source properties/populations, acceleration/propagation mechanisms, and particle physics issues at high energies [73]. After the first results at the Large Hadron Collider (LHC), the hypothetical "particle physics" origin of the knee, due to possible changes in the hadronic crosssections at high energy, is clearly disfavored, confirming that it is a genuine property of the CR spectrum itself [73]. It remains still unsolved whether the (dominant) origin of the knee is due to the reach of the maximum energy achievable at the galactic sources or to limits in the galactic confinement processes. In both cases a rigidity dependent sequence of knees in each single element spectrum is the most probable scenario [74]. For the analysis of the CR flux, direct measurements carried on space or stratospheric balloons actually give the best performance in terms of both energy resolution and charge identification. However, due to their limited acceptance and the steeply falling fluxes, they do not give clear information on the steepening of various elements nor on the knee of each species or of the all-particle spectrum itself.

The measurement of the spectra of the different nuclear components in the knee region will also help to interpret the galactic component of the neutrino diffuse emission which will be accurately measured by IceCube and Km3Net (photons are likely to be attenuated by the interstellar radiation field). This will be very useful to understand if the CR knee is the same in the whole Galaxy hence to fully understand its nature [75].

Chemical composition is shown to evolve towards heavier nuclei, with helium approaching hydrogen at energies of about $10-20 \mathrm{TeV}$ [76]. It is then mandatory to explore the energy region up to a few $\mathrm{PeV}$ with high precision direct measurements to study the energy spectra of each individual nuclear species, to detect any possible hardening of the spectral indexes and to set mass composition below the knee of the allparticle spectrum. A detection of the steepening of each single species and the ensuing explanation of the all-particle knee would be a crucial result in understanding galactic $\mathrm{CR}$ physics, also serving as fundamental input to the study of the extra-galactic component.

Direct measurement of the all-particle spectrum up to the knee and study of the $p$ and $\mathrm{He}$ components up to $\mathrm{PeV}$ energies are needed to understand the origin of the knee, one of the outstanding problems in $\mathrm{CR}$

CR transport in the heliosphere An improved understanding of transport mechanisms inside the heliosphere translates to an improvement on the uncertainties for indirect DM searches and of the measurement of CR interstellar spectra [13]. In recent years, the energetic particle environment has been studied in great detail on Low Earth Orbit, in particular by the PAMELA experiment on polar orbit on Resurs DK1 (2006-2016) and by AMS-02 onboard the ISS (since 2011).

Inside the heliosphere magnetic turbulence and solar wind disturbances affect CRs up to energies of tens of $\mathrm{GeV}$, causing the so-called "solar modulation" 
phenomenon. To determine how the CR flux evolves in response to the changing conditions of the Sun's magnetic activity is a central question in solar physics. A related problem is to determine the properties and occurrence of solar energetic particle (SEP) events. In this respect, multichannel measurements of CR flux variations at various timescales will allow us to gain a deep understanding on heliospheric physics, from predicting solar modulation to characterizing SEP events.

Operating ALADInO on L2, far from the Earth's magnetic field, would greatly improve the study of solar effects on CRs and disentangle these effects in their Local Interstellar Space spectrum.

Multi-particle precision flux measurements up to several $\mathrm{GeV}$ and far from the Earth's magnetosphere would provide new valuable information on the CR interactions with the heliospheric plasma and significantly improve the accuracy of some DM indirect searches.

\subsection{Observational requirements}

From the ambitious particle astrophysics science program discussed above we derive measurement requirements which we discuss in this section. We review the current experimental status on different measurement channels and discuss the expected sensitivities from ALADInO, whose design concept is presented in the following section 4.

The key physical quantities characterizing the basic properties of CR particles are charge magnitude and charge sign, incoming direction, velocity, momentum, and/or total energy. The instrument must be capable of identifying electrically charged particles with the ability to discriminate between particles and antiparticles over unexplored energies and with orders of magnitude better separation with respect to present experiments. Discrimination between leptons and hadrons, charge measurements, and mass separation will provide full particle identification. An important performance parameter is the collection factor (in units of $\mathrm{m}^{2} \mathrm{sr} \mathrm{yr}$ ), representing the product between exposure time and total acceptance over a given energy range. The measurement requirements depend on the specific observational goals and are outlined in the following.

\subsubsection{Nuclear antimatter}

The detection of anti- ${ }^{4} \mathrm{He}$ or heavier antinuclei would have profound significance in cosmological theories and astrophysics. Conversely, an upper limit on the flux of antinuclei compatible with the range of the expected direct production from CR interaction could be interpreted as a lower limit on the antimatter content in a Universe where normal matter is dominant.

The best experimental limit on the anti-He/He flux ratio is set at $6.9 \times 10^{-8}$ level in a very limited rigidity range (1.6-14 GV) by the BESS experiment [77] in a wider interval $(0.6-600 \mathrm{GV})$ the PAMELA spectrometer has published a limit of $2.67 \times 10^{-7}$ [17]. Hints for anti- ${ }^{3} \mathrm{He}$ and anti- ${ }^{4} \mathrm{He}$ candidates presented by the AMS-02 experiment [21] lie just below the PAMELA upper limit. 
To prove unambiguously the AMS-02 hints of anti-He, or, conversely, to improve the current limits by at least a factor $10^{3}$ requires an identification capability of one antiHe out of $10^{11} \mathrm{Z}=2$ particles over a wide rigidity range. Similar performances are required for $Z>2$ anti-nuclei. Following the analysis of [20], the density of anti-stellar objects in our Galaxy might be approximated by the number of normal matter stars $\left(\approx 10^{11}\right)$ multiplied by the antinuclei-to-nuclei ratio or limit. Using this approach, the upper limit in case of no detection would directly limit the density of antimatter objects in the Milky Way.

Production of anti- ${ }^{3} \mathrm{He}$ can be foreseen also in some DM scenarios or, although strongly suppressed, in the collision of CR with the ISM as discussed in section 3.1.2. ALADInO sensitivity would allow the first study of such a signal.

For antinuclei heavier than anti-He, the current observational limits would be improved at least of 4 orders of magnitude.

\section{REQUIREMENT 1}

A collection factor of $30 \mathrm{~m}^{2} \mathrm{sr}$ yrs is needed to extend the current observational limits for anti- ${ }^{4} \mathrm{He}$ and heavier antinuclei by at least 3 and 4 orders of magnitude, respectively.

\subsubsection{Dark matter}

Positrons and electrons Clear evidence of an unexpected $\mathrm{e}^{+}$excess in CRs, starting at $25 \mathrm{GeV}$, has been reported by PAMELA [81, 82]; accurate measurements by AMS-02 [79-81], have extended the measurement of the positron fraction and the separate $\mathrm{e}^{ \pm}$ fluxes up to $\mathrm{TeV}$ energies. The $\mathrm{e}^{+}$flux exhibits a significant excess over the expected background starting at $25 \mathrm{GeV}$, sharply dropping above $300 \mathrm{GeV}$. This behavior is compatible with a descriptive model requiring a new $\mathrm{e}^{+}$source term that dominates above $100 \mathrm{GeV}$, with a cutoff at $\sim 800 \mathrm{GeV}$ [78].

Different spectral features of the energy spectra of $\mathrm{e}^{+}$and $\mathrm{e}^{-}$in the $200 \mathrm{GeV}$ - multi$\mathrm{TeV}$ energy region are key signatures to separate DM from astrophysical sources or propagation mechanism scenarios. As it can be seen in Fig. 2-right, from the accurate measurement of the falling part of the $\mathrm{e}^{+}$spectrum above $300 \mathrm{GeV}$ it will be possible to test various hypotheses: DM (blue dots), pulsar (red and pink dots), or multiple pulsar (green dots). The accuracy of the current measurements at the $\mathrm{TeV}$ scale, is limited by the statistics available and the energy reach. The supra-TeV $\left(\mathrm{e}^{+}+\mathrm{e}^{-}\right)$spectrum is being accurately measured by ongoing calorimetric experiments (CALET [83], DAMPE [84]): its accuracy will be further improved by forthcoming calorimetric missions (HERD [85]). However, the lack of charge-sign identification of these instruments will not allow for a clear identification of the different spectral features of the $\mathrm{e}^{+}$and $\mathrm{e}^{-}$ fluxes. Conversely, accurate $\mathrm{TeV}$ region spectrometric measurements of the individual $\mathrm{e}^{-}$and $\mathrm{e}^{+}$fluxes at the percent level energies will dramatically restrict the parameter space region for either the DM or the pulsar hypothesis. No project, however, is currently planned to extend the spectrometric measurements by PAMELA and AMS02. ALADInO, taking advantage of a collection factor of $15 \mathrm{~m}^{2} \mathrm{sr}$ yrs. and of a Maximum Detectable Rigidity (MDR) larger than $20 \mathrm{TV}$, would provide a breakthrough advance in this field (see Fig. 2-left). The suppression of the hadronic background would be provided by an hadron rejection larger than $10^{5}$, derived from the combined information of the spectrometer and the calorimeter, together with the 
suppression below $10^{-2}$ of the $\mathrm{e}^{-}$induced background in the $\mathrm{e}^{+}$channel due to charge flip: this will guarantee a background level in the $\mathrm{e}^{+}$channel below $1 \%$ in the $\mathrm{e}^{+}$excess region up to several $\mathrm{TeV}$. The large ALADInO collection factor will guarantee a sample of $\mathrm{e}^{+}$sufficiently large, so that the finite knowledge of the detector efficiencies will dominate the measurement uncertainties at the percent level at the $\mathrm{TeV}$ scale, while the supra-TeV region uncertainties will be dominated by statistical fluctuations of the collected data. In this scenario, the positron excess will be accurately characterized in its whole development from its rise below $100 \mathrm{GeV}$ up to the supra$\mathrm{TeV}$ region where it is expected to drop down to the secondary astrophysical $\mathrm{e}^{+}$ background.

The best limit on $\mathrm{e}^{+}$dipole anisotropy is set by AMS-02 at $1.9 \%$ above $16 \mathrm{GeV}$ [78]. The search for anisotropies in the incoming directions of $\mathrm{e}^{ \pm}$will take advantage of ALADInO's high collection factor, the background rejection capabilities and the instantaneous coverage of almost the full sky, resulting in an expected sensitivity better than $0.2 \%$ in the $\mathrm{e}^{+}$dipole anisotropy above $16 \mathrm{GeV}$. This will reveal novel and unprecedented information, improving the current limits by a factor 10 and probing for the first time $\mathrm{e}^{+}$anisotropies at $\mathrm{TeV}$ energies, possibly providing conclusive information on the origin of high-energy $\mathrm{e}^{ \pm}$.

\section{REQUIREMENT 2}

The measurement of electrons and positrons up to $\sim 5 \mathrm{TeV}$ of energy with a \% accuracy translates into a requirement on the collection factor of $\sim 15 \mathrm{~m}^{2}$ sr yrs. A lepton/hadron separation factor larger than $10^{5}$ is essential to avoid proton contamination. Misidentification of electrons as positrons due to charge confusion must be kept below $10^{-2}$ at all energies to prevent electron-induced background.

Antiproton/proton ratio The most recent measurements of CR antiprotons are made by the AMS-02 experiment [86]. The AMS-02 data show an unexpectedly flat antiprotonto-proton ratio $(\bar{p} / p)$, in the high-energy region between $\sim 60$ and $450 \mathrm{GeV}$, which is at tension with the astrophysical background models based on secondary production of antiprotons from CR collisions with the gas. In fact, background models predict a rapid decrease for the high-energy $\bar{p} / p$ ratio, similarly to the $\mathrm{B} / \mathrm{C}$ ratio. Such a tension hints at
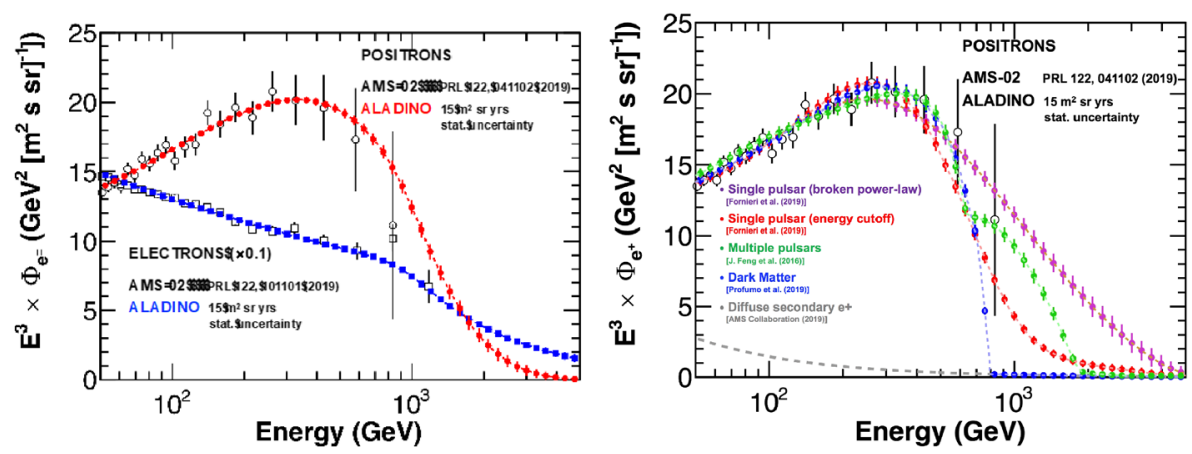

Fig. 2 Left) Projected measurement of ALADInO for $\mathrm{e}^{ \pm}$fluxes in the descriptive model of [78, 79] with a TeV break compatible with observations of [84]. Fluxes are multiplied by $\mathrm{E}^{3}$ and the $\mathrm{e}^{-}$flux scaled down of a factor 10 for display purposes. Right) Projected $\mathrm{e}^{+}$flux measurement in 4 different scenarios invoking astrophysical and DM origin of high-energy $\mathrm{e}^{-}$. The accuracy of ALADInO data in the supra-TeV region will allow to disentangle the dominating source of high-energy $\mathrm{e}^{ \pm}$to be disentangled 
scenarios with extra (DM) sources of high-energy antiprotons though, in contrast to positrons, it could in principle be ascribed to undetected uncertainties in the astrophysical background $[6,9]$. The current $\bar{p} / p$ data favor two classes of DM scenarios, as illustrated in Fig. 3 [87]. In the left panel of the figure, is shown a scenario with $\sim 60 \mathrm{GeV}$ mass DM annihilating into hadronic channels $b \bar{b}$ pairs, giving rise to an antiproton signal at the $0.1-10 \mathrm{GeV}$ energy scale, where the background models underpredict the antiproton flux. In the right panel, the figure shows a scenario with $\sim 10 \mathrm{TeV}$ mass scale DM giving rise to a high-energy excess of antiprotons, from $\sim 100 \mathrm{GeV}$ to $10 \mathrm{TeV}$. In both figures, the gap between the AMS-02 data and the predicted background leaves room for DM annihilation contributions.

Claims for DM evidence have been made in many recent theoretical works, roughly based on these two classes of scenarios [5, 31, 88-91]. From the projected antiproton measurements for ALADInO, shown in Fig. 3, it is clear that the discovery potential for DM spans a large range of mass scales, from $\mathrm{O}(10 \mathrm{GeV})$ to multi-TeV. Regarding the astrophysical background estimation, the situation with antiprotons is highly model dependent. Improved background calculations can relieve either the low-energy or high-energy tensions, but not both tensions at the same time, and not without testable consequences for the high-energy spectra of secondary nuclei (see below). Precision multi-TeV data on secondary nuclei will then be crucial at discriminating among CR propagation scenarios for astrophysical background.

It is also worth noticing that the pulsar scenario considered for the explanation of the positron fraction does not predict any excess in the antiproton flux. Models of extra sources of antiprotons from astrophysical processes involve the hadronic production of secondary CR inside the shockwaves of supernova remnants [59, 92-94]. These models can be definitely tested using secondary nuclei data at multi-TeV energies.

Thanks to its large acceptance, long duration and orbital operation far from the Earth magnetic field, ALADInO will provide more than two orders of magnitude increase in the existing antiproton statistics in the energy range $100 \mathrm{MeV}-5 \mathrm{TeV}$.

\section{REQUIREMENT 3}

Testing the TeV-scale mass DM annihilation requires a collection factor of $\sim 15 \mathrm{~m}^{2}$ sr yrs to extend the antiproton flux measurement by at least one decade in energy. To achieve the needed sensitivity, the antiproton background must be suppressed. This
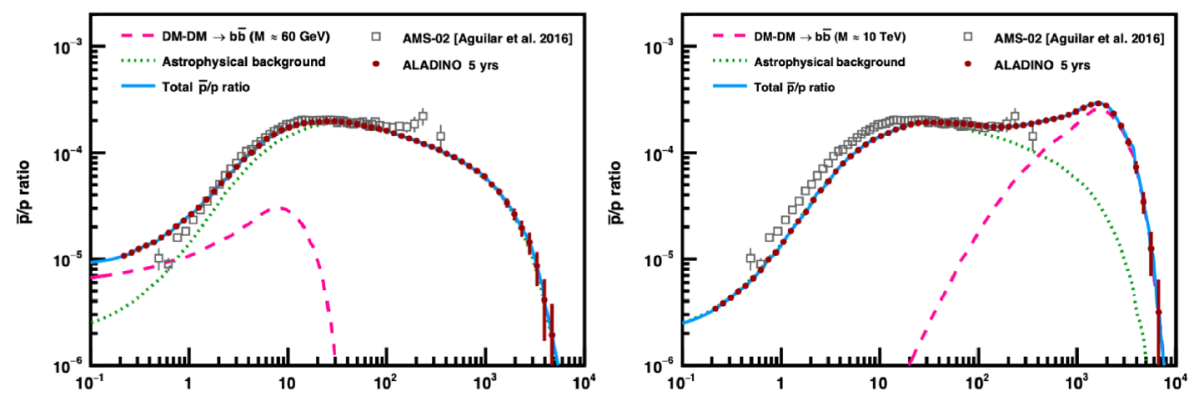

Fig. 3 Antiproton-to-proton ratio as a function of kinetic energy according to standard calculations for the astrophysical background: the plots illustrate the effect of two benchmark models, as discussed in the text. The two scenarios represent $10 \mathrm{TeV}$ (right) and $60 \mathrm{GeV}$ (left) mass DM particles annihilating in b-bbar, from [87]. In both scenarios, projections for ALADInO in 5 years of observation times are shown 
requires a rejection factor of $\sim 10^{5}$ against mis-identified protons and an electron/proton separation of $\sim 10^{4}$.

ANTIDEUTERIUM and ANTIHELIUM In addition to the measurement of the antiproton spectrum, complementary DM searches with antideuteron nuclei in the sub-GeV energy region would benefit from promising DM signals and kinematically suppressed background, thereby offering a potential breakthrough for new physics discoveries [94]. The situation is similar with anti- ${ }^{\mathbf{3}} \mathbf{H e}$ in terms of signal/background ratio, although the absolute signal level is significantly suppressed at increasing mass number [95-97].

The ideal requirement, for these channels, would be to reach the level of astrophysical background, testing any model of DM annihilation that gives rise to a detectable excess above the background. Figure 4 shows flux calculations for antideuteron (left) and anti- ${ }^{3} \mathrm{He}$ (right) as a function of kinetic energy per nucleon, including predictions for the DM models (blue lines) consistent with present antiproton data [89, 98]; an up to date calculation of the astrophysical background is also included, along with its uncertainties [92]. The simulated flux measurements with ALADInO are shown as red markers for the astrophysical background and for the DM signals. The best current upper limits are also shown [77, 99]. Antideuteron searches are and will be performed by AMS-02 and GAPS during the coming years. These instruments combined will only approach $10^{-6}$ flux sensitivity, leaving untested a significant region of parameter space of DM models. The expected AMS-02 sensitivity in 10 years is $\sim 10^{-9}$ for antihelium/ helium ratio at $0.1-50 \mathrm{GeV} / \mathrm{n}$. The dotted lines show the ALADInO sensitivity level, for 10 years of exposure, over its optimal range of detection.

With a predicted sensitivity in 5 years of $\sim 5 \times 10^{-9} \mathrm{part} /\left(\mathrm{m}^{2} \mathrm{sr} \mathrm{s} \mathrm{GeV} / \mathrm{n}\right)$ at the $95 \%$ C.L in the $0.1-1.5 \mathrm{GeV} / \mathrm{n}$ kinetic energy region, ALADInO will probe most models of light and heavy DM particles in the antideuteron channel, and a vast region of parameter space for in the antihelium channel.

\section{REQUIREMENT 4}

The astrophysics background level of secondary CR antinuclei can be reached with $20 \mathrm{~m}^{2} \mathrm{sr}$ yrs of exposure on antideuteron (antihelium) in the $\mathrm{GeV}$ energy region. The optimal energy range to detect DM-induced excesses is $100 \mathrm{MeV} / \mathrm{n}$ - few $\mathrm{GeV} / \mathrm{n}$.
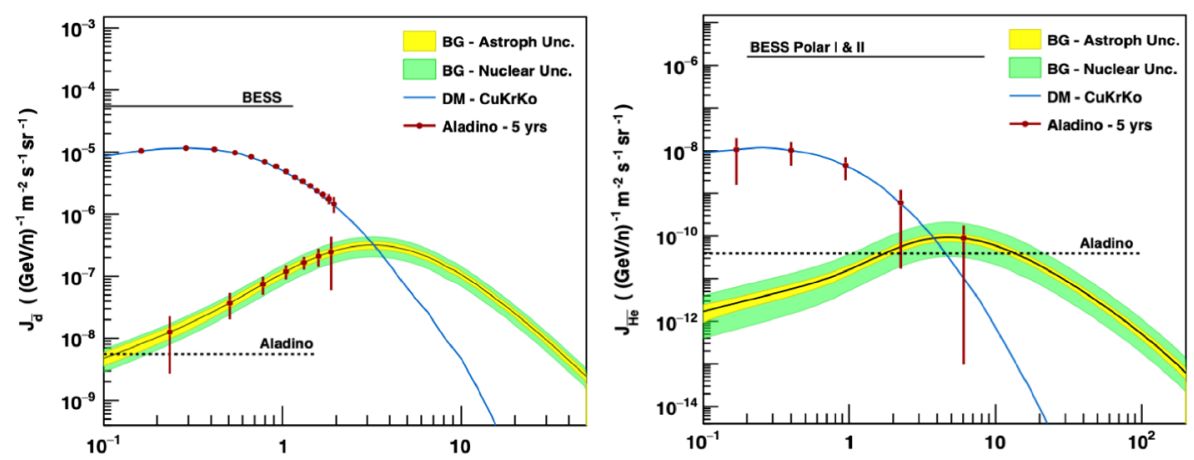

Fig. 4 Antideuteron (left) and anti3He (right) fluxes as a function of kinetic energy per nucleon. See text for description 


\subsubsection{High energy CRs (electrons, protons and nuclei)}

High energy electrons The precise measurement of the supra-TeV $\mathrm{e}^{ \pm}$spectral features could provide relevant information to assess the nature of the observed excess in the positron flux. The measurement of the total $\left(\mathrm{e}^{+}+\mathrm{e}^{-}\right)$flux is insensitive to charge sign identification: it is an interesting observable for both spectrometric and calorimetric space experiments, as well as for ground observatories. The experimental scenario is varied. Indirect ground measurements first revealed, although with large uncertainties, a sudden drop in the $\left(\mathrm{e}^{+}+\mathrm{e}^{-}\right)$flux intensity starting at $1 \mathrm{TeV}$ [100-102]. The drop has been recently confirmed by direct space measurements [103-105] which have studied the $\mathrm{e}^{ \pm}$ spectrum up to $\approx 5 \mathrm{TeV}$. However, no evidence of a break up to $2 \mathrm{TeV}$ is observable in the Fermi-LAT measurement [106] nor from the latest AMS-02 results [78, 79]. Finally, in the 300-1000 GeV energy range, discrepancies in the flux intensities up to $30 \%$ are observed between measurements from different space detectors.

The large statistical uncertainties above the $\mathrm{TeV}$ region and the observed discrepancies below the TeV region add further complexities in the data interpretation of the origin of the spectral break and of the tentative feature observed at $1.4 \mathrm{TeV}$ by DAMPE, which could be explained involving new astrophysical mechanisms or DM related effects [64, 107-114]. Redundant measurements of the $\left(\mathrm{e}^{+}+\mathrm{e}^{-}\right)$energies and spectra are a fundamental requirement to reduce systematic uncertainties. ALADInO, taking advantage of its calorimetric collection factor of $20 \mathrm{~m}^{2} \mathrm{sr}$ yrs. and a hadron rejection capabilities larger than $10^{5}$, will provide a real advance in the field. Below 10 $\mathrm{TeV}$ the hadronic background will be kept below $0.1 \%$. The measurement uncertainty will be consequently dominated by the knowledge of the calorimeter energy scale which, as for the AMS-02 experiment [115], will be improved by at least a factor of 2 to 3 with respect to calorimeter-only instruments by the cross-calibration with the rigidity measurement of the spectrometer. The large collection factor will extend the sensitivity of ALADInO to characterize the spectrum of nearby astrophysical $\mathrm{e}^{ \pm}$sources up to $50 \mathrm{TeV}$, as shown in Fig. 5. In the scenario where the supra-TeV region will also be measured by the CTA ground observatory [116] and the calorimetric space mission HERD [85], ALADInO will provide the most accurate characterization of the $\left(\mathrm{e}^{+}+\mathrm{e}^{-}\right)$ flux at $1 \mathrm{TeV}$ and above, determining with unique precision the only observational feature that is planned to be used by CTA to calibrate its energy scale [117].

REQUIREMENT 5

The measurement of $\left(\mathrm{e}^{+}+\mathrm{e}^{-}\right)$up to $\sim 50 \mathrm{TeV}$ requires a collection factor of $\sim 20 \mathrm{~m}^{2} \mathrm{sr}$ yrs. A lepton/hadron separation larger than $\sim 10^{5}$ is essential to avoid proton contamination. The cross-calibration of the calorimeter energy scale with the rigidity spectrometer measurement will allow to determine spectral features with an accuracy better than $1 \%$.

CR PROTONS AND NUCLEI UP TO $50 \mathrm{TEV} / \mathrm{n}$ Recently, measurements of CR proton and helium fluxes performed by PAMELA and AMS-02 revealed unexpected anomalies in the high-energy range of CR spectra [118]. In particular, the proton spectrum from $\sim 10 \mathrm{GeV}$ up to $1.8 \mathrm{TeV}$ of energy was reported to be appreciably harder than the helium spectrum while, in the energy spectra of both species, a remarkable change of the spectral index (or spectral hardening) was observed to occur at about $200 \mathrm{GeV}$ of energy [68, 119-121]. 

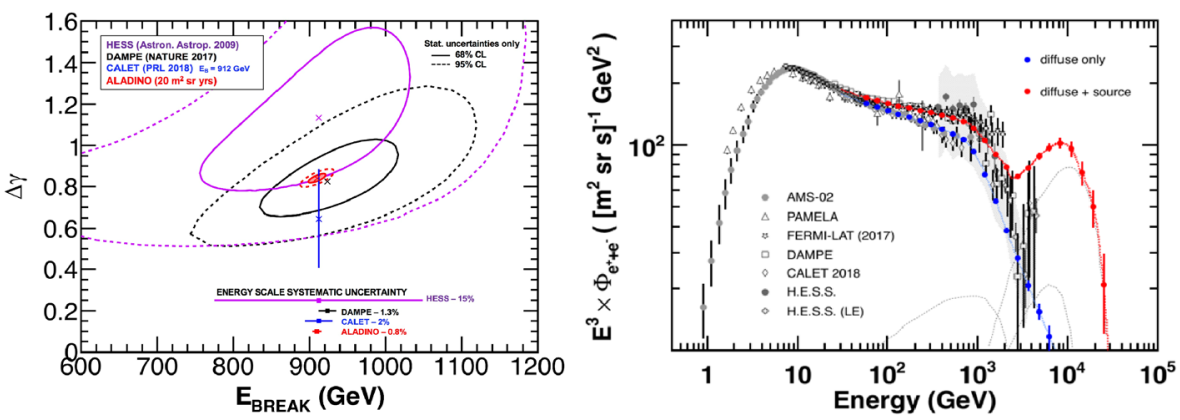

Fig. 5 Left: Sensitivity for the measurement of $\left(\mathrm{e}^{+}+\mathrm{e}^{-}\right)$flux break energy $\left(\mathrm{E}_{\mathrm{BREAK}}\right)$ and index variation $(\Delta \gamma)$ based on the parametrization used in [103]. Right: Projected $\left(\mathrm{e}^{+}+\mathrm{e}^{-}\right)$flux measurement with ALADInO in a scenario with a diffuse flux and with a contribution of a nearby astrophysical source in the supra-TeV energy range. Fluxes are multiplied by $\mathrm{E}^{3}$ for display purposes

More recently, $Z>2$ measurements from AMS-02 reported that the hardening effect is present in essentially all charged elements of the cosmic radiation, including primary, secondary fluxes, and even in secondary/primary ratios $[69,119,120,121]$. Explanations for the CR spectral anomalies may involve the interplay of various physics processes at work such as, e.g., shock (re)acceleration mechanisms, nonlinear/ inhomogeneous diffusive propagation, or stochasticity effects related to nearby $\mathrm{CR}$ sources. All these mechanisms are currently subjected to extensive theoretical research: observationally, the measurement of multichannel CR flux data at the multi-TeV energy scale is a highly-demanded experimental effort [65, 122, 123]. Measurements of primary (e.g. C-N-O) and especially secondary (Li-Be-B) nuclei in the currently unexplored $\sim 1-50 \mathrm{TeV} / \mathrm{n}$ energy range are also essential for DM searches. The different physics models for the CR spectral anomalies give distinct predictions for the secondary/primary ratios at high energies [118]. Thus, measurements such as the $\mathrm{B} / \mathrm{C}$ ratio at multi-TeV energies will give an invaluable asset to calibrate the astrophysical background of antiprotons and antinuclei for DM searches.

Based on these considerations, the scientific requirement for ALADInO is to provide an accurate spectrometric measurement up to $20 \mathrm{TV}(10 \mathrm{TeV} / \mathrm{n})$, with a charge resolution of $\delta Z \sim 0.15$ (at least for $Z>2$ ), based on multiple charge measurements to ensure a background-free and fragmentation-free detection of rarer elements such as LiBe-B. The $100 \mathrm{TeV} / \mathrm{n}$ energy scale can be realistically achieved by means of calorimetric measurements. In both detection modes, a collection factor of about $20 \mathrm{~m}^{2} \mathrm{sr}$ yrs. is needed to ensure statistics up to the highest measurable energy region. The simultaneous measurement of the energy/rigidity performed by the spectrometric and calorimetric modes will allow for the in-flight cross-calibration of the absolute energy scale, thus providing reliable measurements of CR energy spectra at unexplored regions.

An example of the expected B/C measurement from ALADInO is shown in Fig. 6. The figure shows two benchmark models for the $\mathrm{B} / \mathrm{C}$ ratio that are currently unresolved by the existing data [123].

REQUIREMENT 6

Measuring nuclear fluxes up to $50 \mathrm{TeV} / \mathrm{n}$ will be

possible thanks to the large calorimetric acceptance and the redundant 


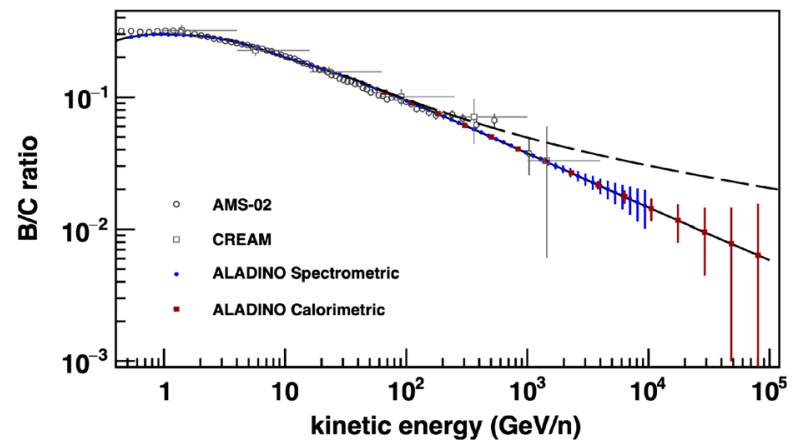

Fig. 6 ALADInO expectation for the B/C flux ratio, for an exposure of $20 \mathrm{~m}^{2}$ sr yrs. Two benchmark models for the $\mathrm{B} / \mathrm{C}$ are shown, both consistent with the existing data. The simulations are shown for both the spectrometric and calorimetric modes

measurements of particle charge.

A collection factor of $20 \mathrm{~m}^{2} \mathrm{sr}$ yrs is required for the measurement of $2<\mathrm{Z}<8$ species.

Reaching the knees Direct measurements could hardly reach energies of hundreds of $\mathrm{TeV}$ due to the limited detector acceptance: it follows that no clear information exists on the knees of the individual Z-resolved CR elemental species. Conversely, groundbased arrays have larger exposures but they are affected by serious uncertainties on the energy scale and very limited particle identification capabilities. Current (planned) missions such as CALET or DAMPE (HERD) are extending the measurements towards $100 \mathrm{TeV}$. ALADInO in the calorimetric mode would exhibit a collecting factor of $20 \mathrm{~m}^{2} \mathrm{sr}$ yrs.; the proton and helium spectra will be measured individually up to the PeV energy scale. This would be the first direct evidence and determination of the CR knees in the single elemental components.

\section{REQUIREMENT 7}

A collection factor of at least $20 \mathrm{~m}^{2} \mathrm{sr}$ yrs is required to measure the proton and $\mathrm{He}$ flux up to $1 \mathrm{PeV} / \mathrm{n}$ of energy.

$\mathrm{Cr}$ transport in the HELIOSPHERE The study of long-term solar modulation of CRs requires measurements of particle fluxes over monthly, weekly, and even daily bases. This can be done with an acceptance of at least $\sim 0.5 \mathrm{~m}^{2} \mathrm{sr}$, in the $\sim 0.1-$ $50 \mathrm{GeV} / \mathrm{n}$ energy range, on an orbit with low-rigidity cutoff like on L2. The stability of the detector performance with time is an important concern for this investigation. With these requirements, and the possibility to monitor the CR fluxes over a significant fraction of the 11-year solar cycle, the ALADInO mission will enable the investigation of various subjects in heliophysics. This also includes the precise measurements of the flux, composition and arrival time of solar energetic particle events, in particular during rare high-energy events associated with solar flares or Coronal Mass Ejections, making ALADInO a privileged observatory of solar activity from deep space orbit.

\section{REQUIREMENT 8}

Provide daily measurements of proton and He fluxes at 0.1-50 GeV/n of energy,

The mission should be located on a deep space orbit with an acceptance of at least $0.5 \mathrm{~m}^{2} \mathrm{sr}$. 


\section{Measurement concept}

A possible ALADInO detector configuration matching the measurements requirements discussed in the previous section is briefly presented here. The detector is equipped with a magnetic spectrometer for CR charge sign identification and rigidity measurement up to a Maximum Detectable Rigidity (MDR) larger than $20 \mathrm{TV}$, a 3D imaging calorimeter, with high energy resolution (depth $\sim 60 \mathrm{X}_{0}, \sim 4 \lambda_{\mathrm{I}}$ ) for precise measurement of the $\mathrm{e}^{ \pm}$and hadronic spectral features above the spectrometer MDR, and a Time of Flight with $O(\%)$ resolution in the velocity measurement for efficient deuteron/ proton separation.

The detector concept exploits the isotropy of the cosmic-ray flux, maximizing the collection power within a classical toroidal magnetic configuration: particle detection and identification is based on well-established and reliable space qualified technologies.

The basic ALADInO payload is based on three key elements:

1. A High Temperature Superconducting (HTS) magnetic spectrometer (SMS) to measure the particle rigidity, charge magnitude and sign, with a maximum detectable rigidity exceeding $20 \mathrm{TV}$ and an acceptance $>10 \mathrm{~m}^{2} \mathrm{sr}\left(\sim 3 \mathrm{~m}^{2} \mathrm{sr}\right.$ in combination with the calorimeter);

2. Time of Flight (ToF) system to measure the particle velocity and charge magnitude;

3. a large acceptance $\left(\sim 9 \mathrm{~m}^{2} \mathrm{sr}\right) 3 \mathrm{D}$ imaging calorimeter (CALO) to measure particle energy and separate the rare electromagnetic component $\left(\mathrm{e}^{ \pm}, \gamma\right)$ from the overwhelming hadronic component of cosmic rays.

The ToF and the CALO also provide signals to trigger the start of data acquisition on minimum ionizing and showering particles respectively.

A possible design of the ALADInO detector that maximizes the acceptance while keeping the overall payload size compact is presented in Fig. 7. The detector has a cylindrical shape with a diameter of $440 \mathrm{~cm}$ and $200 \mathrm{~cm}$ in length, designed to fit within the fairing volume of an Ariane 6 class launcher. This design fully exploits the CR isotropy: particles are collected over a wide solid angle on the lateral surface of the cylinder. The axial symmetric configuration of the detectors guarantees a uniform response of detectors independent of the particle arrival direction.

It should be noted that ALADInO could also detect $\gamma$-rays in the $\mathrm{GeV}-\mathrm{TeV}$ energy range: this capability would allow a variety of studies in high energy astrophysics and fundamental physics, including indirect DM searches. Thanks to its large acceptance and to the all-sky coverage, ALADInO can monitor high-energy $\gamma$-ray sources and detect transient events as well. These observations would fit in the framework of a multi-messenger and multi-wavelength strategy of the next generation of space-borne and ground-based instruments (e.g. gravitational wave observatories, both groundbased and space-based, as well as high energy telescopes spanning from keV-to $\mathrm{TeV}$ ).

The ALADInO concept exploits two innovative techniques:

- the use of a superconducting magnet based on a high temperature lightweight superconductor material. This design allows to reach an intense magnetic field over 

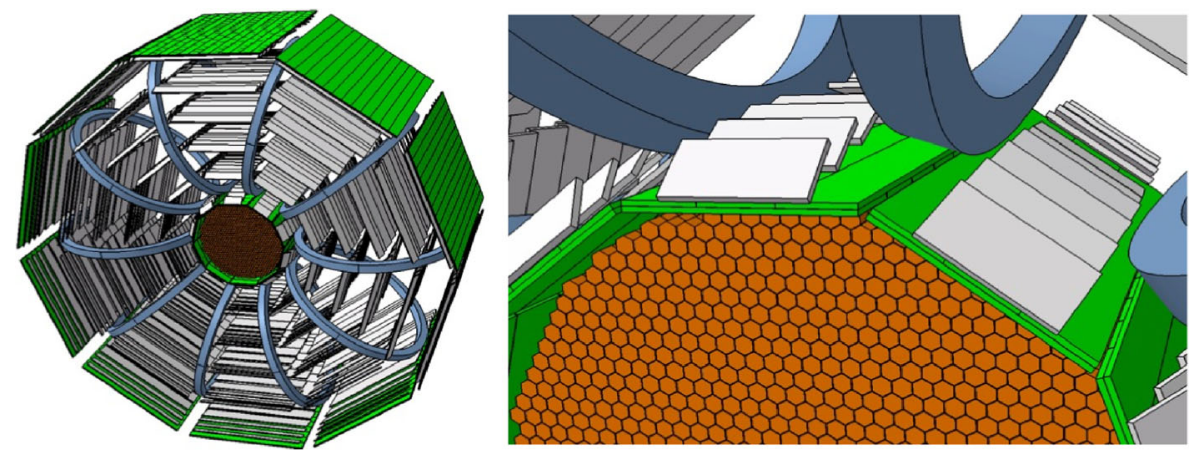

Fig. 7 Left: rendering of the ALADInO detector. The core of the apparatus is a cylindrical calorimeter, (dark orange). Ten circular magnetic coils (blue), surround the calorimeter. A silicon tracking system (gray), is arranged in six layers each composed of several modules. Two time-of-flight layers segmented in paddles (green), are located outside the outer tracker layer and below the innermost tracker layer. Right: detail of the central part of the apparatus; the segmentation of the calorimeter in (x-y) view is clearly visible, as well as the time of flight inner layers placed between the calorimeter surface and the first tracker layer. The adaptive orientation of the tracker sensors is set to maximize the trajectory measurement resolution

the large volume of the spectrometer. Thanks to the excellent spatial resolution of silicon strip detectors/pixel detectors the maximum detectable rigidity of the spectrometer will exceed $20 \mathrm{TV}$;

- the use of a cylindrical calorimeter made of a 3D mesh of small hexagonal prismshaped scintillating LYSO crystals. It ensures a nearly isotropic response to particles entering from different directions maximizing the detector acceptance. The highly segmented design together with the depth of $\sim 60 \mathrm{X}_{0}$ allows a good energy resolution, also for hadronic particles, and the required particle identification capability, independent of the particle incoming direction.

The ToF and silicon tracker detectors are based on the successful design used in the AMS [124, 125] and the PAMELA experiment [126, 127].

The main characteristics of the ALADINO experiment are summarized in Table 1. A short description of the different subsystems is presented in the following sections.

\subsection{ToF}

The proposed ALADInO ToF system is designed to perform a precise measurement of the particle velocity and arrival direction based on the time-of-flight technique, to provide a redundant measurement of the particle charge $(Z)$ and to deliver the signal for the charged particle trigger in the Data Acquisition System.

The ToF inherits its concept from the PAMELA and AMS missions: bars of thick plastic scintillators are placed before and after the tracking system running in orthogonal directions over two staggered layers to provide $x-y$ hodoscopic information vetoing charged particles background in the photon trigger. In a benchmark design, the system is made of four layers of $0.8 \mathrm{~cm}$ thick plastic scintillators, placed in pairs before and after the tracker. Counters can be read out on each end by Photomultipliers (PMT) (as in the AMS or PAMELA detectors) or by Silicon Photomultipliers (SiPM), insensitive to magnetic fields. 
Table 1 Key performance parameters of the ALADINO apparatus

\begin{tabular}{ll}
\hline Calorimeter acceptance & $\sim 9 \mathrm{~m}^{2} \mathrm{sr}$ \\
\hline Spectrometer acceptance & $>10 \mathrm{~m}^{2} \mathrm{sr}\left(\sim 3 \mathrm{~m}^{2} \mathrm{sr}\right.$ w/i CALO) \\
Spectrometer Maximum Detectable Rigidity (MDR) & $>20 \mathrm{TV}$ \\
Calorimeter depth & $61 \mathrm{X}_{0}, 3.5 \lambda_{\mathrm{I}}$ \\
Calorimeter energy resolution & $25 \%$ to $35 \%$ (for nuclei) \\
& $2 \%$ for electrons and positrons) \\
Calorimeter e/p rejection power & $>10^{5}$ \\
Time of Flight measurement resolution & $\sim 100 \mathrm{ps}$ \\
High energy $\gamma$-ray acceptance (Calorimeter) & $\sim 9 \mathrm{~m}^{2} \mathrm{sr}$ \\
Low energy $\gamma$-ray acceptance (Tracker) & $\sim 0.5 \mathrm{~m}^{2} \mathrm{sr}$ \\
$\gamma$-ray Point Spread Function & $<0.5 \mathrm{deg}$ \\
\hline
\end{tabular}

The expected time resolution is of about $100 \mathrm{ps,} \mathrm{which} \mathrm{is} \mathrm{sufficient} \mathrm{to} \mathrm{discriminate}$ anti-deuterons from anti-protons at kinetic energies up to a few GeVs.

\subsection{Calorimeter}

Based on the CaloCube R\&D project [128], the calorimeter consists of a 3D mesh of small LYSO scintillating crystals arranged to form a nearly regular cylinder as shown in Fig. 8. The front view of the calorimeter inside the ALADInO experiment is shown in Fig. 7.

The chosen geometry is the most effective compromise between a fully isotropic calorimeter, i.e. a sphere with uniform response to particles over its entire surface, and the need to use part of the field of view for electronics/mechanical structures and services for the payload.

Single elements are shaped as regular hexagonal prisms and arranged to completely avoid escape planes, i.e., particle trajectories completely sneaking in between the calorimeter gaps. This is particularly relevant for energy resolution. Thanks to the adopted geometry, relatively large gaps $(8 \mathrm{~mm})$ can be then left between crystals increasing the total acceptance, at equal mass, without a significant loss of energy resolution.
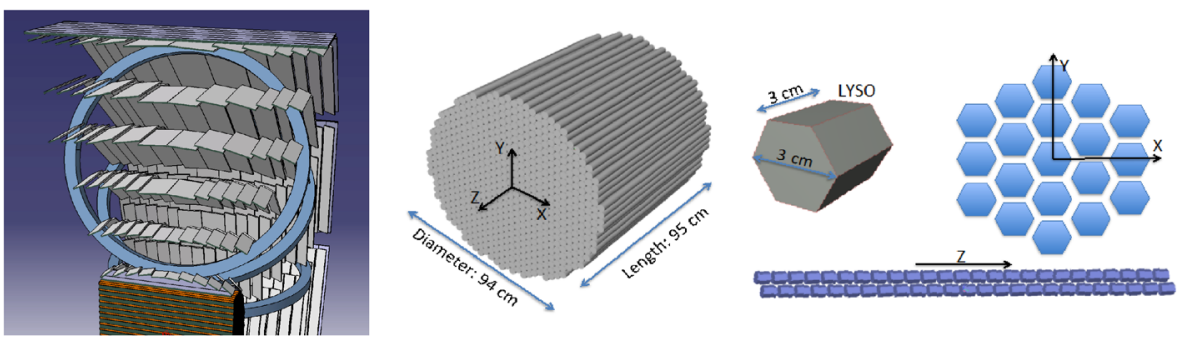

Fig. 8 Left: silicon ladder orientation devised for ALADInO. Right: overall assembly of the hexagonalprismshape crystals and design of two adjacent strings of crystals - crystals are staggered by a half-length to avoid dead space 
The crystals are made of Cerium-doped Lutetium Yttrium Orthosilicate (LYSO). LYSO is a dense, bright inorganic scintillator, which is nowadays commercially available, being industrially produced by several companies for high-precision Positron-Emission Tomography. Its features make it a competitive material for compact calorimeters. It is rather insensitive to temperature changes and thus suitable for highprecision calorimetry in the harsh space environment. Following the design of Fig. 8, and assuming a mass envelope of $2000 \mathrm{~kg}$, it can be shown that a calorimeter depth along the diameter corresponding to $3.5 \lambda_{\mathrm{I}}$ or to $61 \mathrm{X}_{0}$, and a geometric factor of $\sim 9 \mathrm{~m}^{2} \mathrm{sr}$ can be achieved.

The calorimeter performances have been studied using the GEANT4 package [129, 130]. The full geometry was implemented, including the carbon fibre support structure in between crystals. Preliminary results show that an energy resolution of $2 \%$ for electrons and $25 \%$ for protons can be achieved. The latter is valid for well-contained events, selected at the cost of reducing the effective geometric factor (i.e. the geometric factor times the selection efficiency): for a $1 \mathrm{TeV}$ proton it corresponds to $1 \mathrm{~m}^{2} \mathrm{sr}$. By loosening the containment criterion, the resolution reaches $35 \%$ at $5 \mathrm{~m}^{2} \mathrm{sr}$. The electron/proton rejection factor is expected to be better than $10^{5}$, since the CaloCube project achieves this performance while being much shallower than ALADInO and with a similar detector segmentation.

TIC - Tracker in calorimeter the calorimeter layout is focused on optimizing the detection of charged CRs, but, in a general multipurpose experiment in space, the detection and tracking of $\gamma$-rays plays also an important role. The pair-conversion telescopes used by the Fermi-LAT [131] and DAMPE [84] experiments exploit the $\gamma$ conversion in tungsten layers to track the $\mathrm{e}^{+} / \mathrm{e}^{-}$pair in $\mathrm{Si}$ detectors and reconstruct the incidence direction of the original photon. Pairing such a device with the calorimeter would however induce ion fragmentation in tungsten, reducing the charge identification performance; also, the lever arm required for obtaining good tracking performance would reduce the overall geometric factor of the whole detector. All these drawbacks are solved by the TIC approach [132], which uses the calorimeter itself as active pairconversion material in place of the tungsten layers. The photon trajectory is then reconstructed by sampling the lateral development of the induced EM shower at different calorimeter depths by Si microstrip layers intertwined with the external crystal layers of the calorimeter. Different TIC geometries have been investigated using simulations [133], leading to an angular resolution (Point Spread Function, PSF) of the order of $0.5 \mathrm{deg}$ for vertical particles above $10 \mathrm{GeV}$, improving with respect to the performances of Fermi-LAT [134].

\subsection{Spectrometer}

As previously stated, the physics requirements for the ALADInO spectrometer mandate an average MDR larger than $20 \mathrm{TV}$ over a large acceptance. A possible design capable of accommodating such targets must rely on:

- a high-intensity magnetic field generated by a superconducting magnet system surrounding the calorimeter. By choosing a toroidal field configuration it is 
possible to achieve the required bending power over a large acceptance, while also maintaining a theoretical null magnetic momentum;

- a tracking system, composed of silicon detector layers, to provide a measurement of the particle trajectory into the magnet field. Each of the detector layers is divided in modules and arranged cylindrically around the calorimeter;

A proposed overall arrangement of the magnet coils and of the tracking system can be seen in Fig. 8.

\subsubsection{Magnet}

Superconducting magnets (SM) allows for high momentum resolution at the TV scale and over large detection surfaces. The main requirements of SMs for space applications are: (i) low mass budget, i.e. high stored energy to mass ratio; (ii) low power consumption, i.e. efficient cryogenics; (iii) very high stability. The first requirement is obtained by using low density materials and high current density.

Stability and helium cryogenics have been the major problems which have hindered the use of SM technology in space, so far. Stability is the capability of a SM to sustain a sudden energy release without quenching. It is a sensitive issue in designing SMs operating at liquid helium temperature: the problem became less important increasing the operational temperature, due to the cubic temperature dependence of superconductor specific heat.

During the last decades, technological advances have made possible to envisage solutions for space magnets based on high-temperature superconductors (HTS). HTS magnets are operable at temperatures up to $40 \mathrm{~K}$ with the double benefit of solving the problems related to stability and avoiding cryogenics based on liquid He. Among HTS, ReBCO (Rare Earths-Barium Cuprates) tapes are particularly promising for space applications. At present, commercial ReBCO can be operated at $400 \mathrm{~A} / \mathrm{mm}^{2}$ at $30 \mathrm{~K}$ in a $3 \mathrm{~T}$ field. Recent developments indicate that the current density can be pushed to $2000 \mathrm{~A} / \mathrm{mm}^{2}$ in the next years [135]. The use of magnesium diboride wires, despite its poorer current properties, is also a possible option, due to the low average mass density $[136,137]$.

Quench protection techniques are rapidly evolving towards fast and safe procedures guaranteeing magnet integrity even in critical conditions [138].

A toroidal configuration is proposed for the magnet of ALADInO. Advantages and limitations of this solution were discussed within the SR2S project, with the purpose of shielding astronauts from space radiation [139, 140, 135]. More recently, the concept and the technology for an HTS magnet to be used in a space spectrometer have been developed within the HDMS project [141]. HDMS has designed a compact toroidal spectrometer made of ReBCO tape and producing a peak magnetic field of $11.9 \mathrm{~T}$ at an operating temperature of $20 \mathrm{~K}$. Notably, the ultimate goal of HDMS is the fabrication and the operation of a demonstrator coil, to be completed by 2021 [142].

A toroidal configuration guarantees a large geometric acceptance, confining the field within the coils and minimizing the dipole moment. Low density structural materials (Aluminum and Titanium alloys, $\mathrm{Al}-\mathrm{B}_{4} \mathrm{C}$ and $\mathrm{Al}-\mathrm{Al}_{2} \mathrm{O}_{3}$ cermets, aramid fibers) will be used to counter the Lorentz forces. It is worth noting that one of the components of ReBCO tapes is hastelloy, therefore the conductor will contribute to the magnet mechanical structure. 
Bending power the coil number and shape (round, racetrack, D-shaped) will be optimized to maximize the detector performance. Figure 9 shows a possible magnet configuration having $4.3 \mathrm{~m}$ overall diameter dimension with a $1 \mathrm{~m}$ inner bore to host the calorimeter.

With 4.4 MA-turns, the average field on the tracker is $0.8 \mathrm{~T}$ providing a $1.1 \mathrm{~T} \cdot \mathrm{m}$ bending power. The mass of the magnet, with the present technology and including the mechanical structure, is $1200 \mathrm{~kg}$. We estimate that the superconductor technology evolution and a targeted R\&D will allow a mass reduction to less than $900 \mathrm{~kg}$.

Cooling a superconducting magnet operating at $40 \mathrm{~K}$ in deep space could be passively cooled: it would, however, require large area radiators. Therefore, the use of cryocoolers appears to be the favorable choice.

Most of the heat flux from the Sun is passively intercepted by an umbrella-type shield made of V-groove layers [143]. The temperature on the dark side of the sunshield can be estimated as high as $60 \mathrm{~K}$, with a residual heat flux density in the region of $80 \mathrm{~mW} / \mathrm{m}^{2}$. The heat flow can be directed to the external ToF surface to maintain the ToF at its working temperature $(250 \mathrm{~K})$.

The superconducting coils, operating at $30-40 \mathrm{~K}$, are subjected to multi-directional thermal radiation from the calorimeter, ToF, and tracker (at 230-250 K). Therefore, each coil must be surrounded by two thermal shields. The external one, on the detector side, will be actively maintained at $80 \mathrm{~K}$ by a cryocooler and the second one, thermally passive, will be at a temperature close to the magnet working temperature.

The $80 \mathrm{~K}$ shields will be thermally connected with the central thermal link by pulsating heat pipes, to maximize the efficiency/mass ratio [144]. The $80 \mathrm{~K}$ thermal shield will be composed of a light structure supporting a cryogenic multi-layer insulation (MLI). The maximum radiation power to be extracted to maintain the shield at $80 \mathrm{~K}$ is of the order of $50 \mathrm{~W}$. This heat load will be absorbed by the first stage of the cryocooler (see Fig. 10). The second shield will be also composed of 10 to 20 layers of MLI and will be directly wrapped around the coils. From $80 \mathrm{~K}$, the MLI performance is

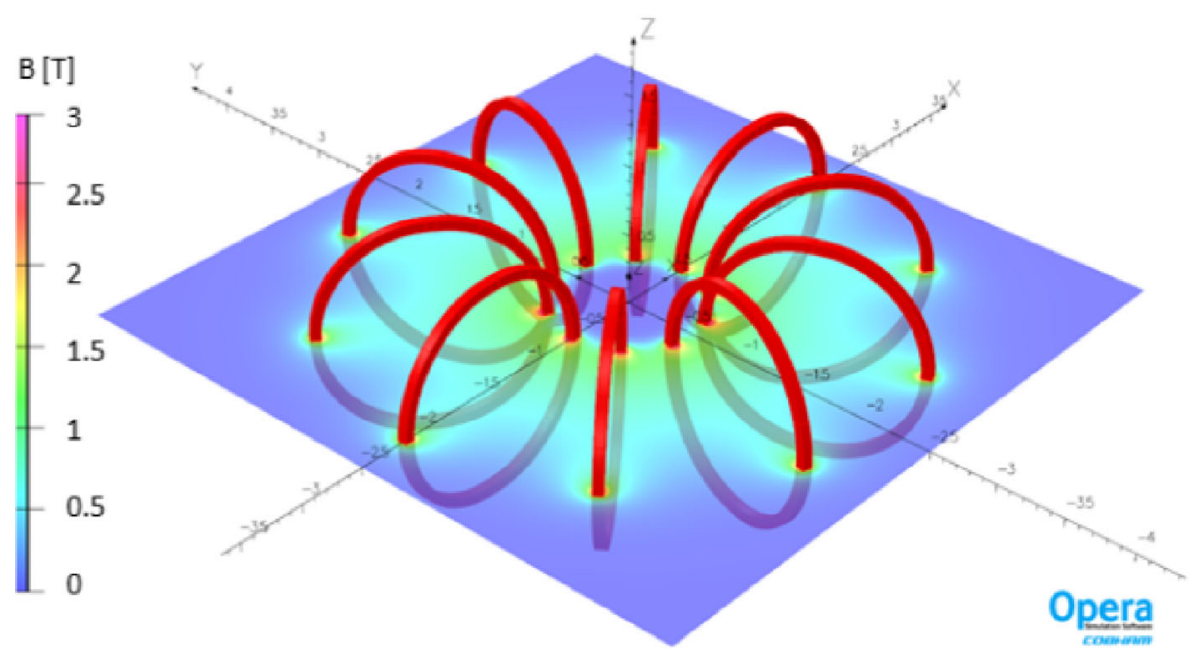

Fig. 9 Scheme of a possible magnet configuration with its field map 
about $0.1 \mathrm{~W} / \mathrm{m}^{2}$ which represents a heat load of $2 \mathrm{~W}$ on the entire coils system at the operational temperature. Mechanical supports will be necessary to maintain the entire assembly together.

Studies are in progress to reduce the tracker and calorimeter operational temperature below $230 \mathrm{~K}$ and consequently to reduce the heat load on the coils.

\subsubsection{Tracker}

Tracking with a $\mathrm{O}(\mu \mathrm{m})$ precision could be easily achieved in ALADInO using doubleside silicon microstrip detectors. Originally developed in the ' 80 s for micro-vertex detectors in the High Energy Physics experiments at colliders, the precursor flight of the AMS experiment on the shuttle Discovery in 1998 has first demonstrated that thin, $\mathrm{O}(300 \mu \mathrm{m})$, silicon microstrip detectors can be successfully operated for tracking in space. In addition to precision measurement of particle coordinates, silicon microstrip detectors are lightweight, minimizing the material along the particle trajectory, thus reducing the probability of particles scattering in the detector material. The absence of consumables and relatively low operating voltages $(\sim 70 \mathrm{~V})$ makes silicon trackers an ideal tracking detector in space, both in calorimetric experiments (AGILE, Fermi, DAMPE) or magnetic spectrometers (PAMELA, AMS-02). Several tens of $\mathrm{m}^{2}$ of silicon detectors are currently operating successfully in space in long-term missions. By coupling the silicon device to a high dynamic range front-end electronics, it is possible to measure the particle charge with high accuracy by looking at the amplitude of the total energy deposited, which is proportional to $\mathrm{Z}^{2}$.

The design of the ALADInO tracking system benefits from the experience of the AMS and PAMELA experiments. By using capacitive charge coupling with floating strips it is possible to get optimal spatial resolution with a reduced number of readout channels, reaching the desired $\mathrm{O}(\mu \mathrm{m})$ accuracy with a reasonable readout pitch of $\sim 100 \mu \mathrm{m}$.

In its baseline design the tracker it is composed of 7540 sensors, $95 \times 95 \mathrm{~mm}^{2}$ each, for a total detector surface of about $68 \mathrm{~m}^{2}$. The silicon sensors are daisy-chained for readout

Side cross-sectional view

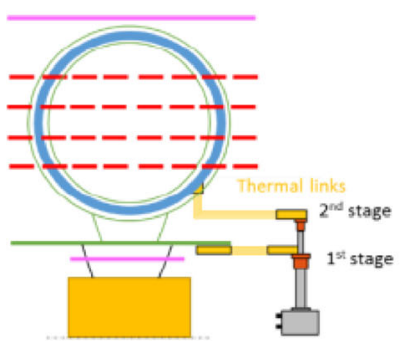

ToF + trigger Cryocooler
Top cross-sectional view

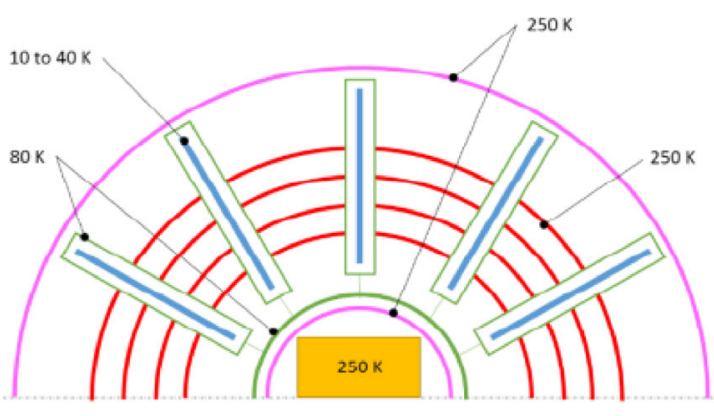

Tracker Cryogenic shield + thermal link Calorimeter + trigger

Fig. 10 Conceptual cryogenic design 
and biasing into modules, made of a variable number of sensors (from 2 to 7 ) to match the size of the different tracker planes. To reduce the material in the tracking volume, frontend electronics are usually located at the end of the modules. For the bending coordinate strips, the daisy chain is then provided through direct bonding from sensor to sensor, while for the transversal non-ben ding strips a metalized film running over the module length is used to connect to the corresponding front-end electronics. The proposed ALADInO design currently features a total of 1400 modules, with length up to seven silicon detectors, which are arranged on six planes surrounding the calorimeter as visible in Fig. 7. The modules will be mounted on light stiff support planes based on the experience of the AMS-02 silicon tracker. Each module will have about 1500 read-out channels.

The readout pitch, together with low noise front-end electronics will ensure a position resolution of about $3 \mu \mathrm{m}$ for normal particle incidence: a similar result has already been obtained with the PAMELA short modules [127] while AMS-02 has demonstrated the possibility to use long detector modules (up to $60 \mathrm{~cm}$ ) with a resolution better than $10 \mu \mathrm{m}$. With a moderate R\&D activity to optimize the readout electronics, it should be possible to obtain the design value of $3 \mu \mathrm{m}$ on long detector modules, with a limited power consumption ( $0.2 \mathrm{~mW}$ per channel [140]).

The dynamic range of the front-end will extend such as to reach at least the Oxygen signal amplitude without saturation, similar to the performance shown by the AMS-02 tracker electronics [144]. In order to fully exploit the intrinsic resolution of the detectors and considering the worsening of the resolution with the track incident angle, a special ladder orientation is considered, as shown in the left panel [142] of Fig. 8.

\subsubsection{Spectrometer performance}

The Maximum Detectable Rigidity (MDR) of such a spectrometer has been estimated through a particle tracking Monte Carlo simulation including the magnetic field configuration and the tracking system geometry. Depending on the particle arrival direction the MDR values obtained range between 10 and $35 \mathrm{TV}$ with a mean value larger than $20 \mathrm{TV}$ : from AMS-02 and PAMELA experience we know that charge sign separation for antiparticle/particle reliable identification, purely based on the magnetic spectrometer, is possible up to $\sim \mathrm{MDR} / 4$ in rigidity. That means that ALADInO experiment can measure antiprotons up to $5 \mathrm{TeV}$, namely one order of magnitude higher than the available results. For the positrons, given the precise particle energy measurement provided by the calorimeter, the charge sign identification will be reliable up to $\sim \mathrm{MDR} / 2$, that is in the $10 \mathrm{TV}$ range, again more than one order of magnitude in energy higher than the data available.

Technology developments During the next years we expect to improve the existing technologies discussed above. At the same time, we are investigating and performing R\&D on new technologies which could replace those already existing. The following gives a sample list.

Silicon photomultipliers (SiPMs) SiPMs can replace traditional photomultipliers for photodetection applications in space. SiPMs are in fact more robust, can be operated in external magnetic fields with low power consumption $(<10 \mathrm{~mW} /$ channel [143]) and without requiring high-voltage supply while providing similar timing performances 
[145]. Devices with microcell size smaller than $30 \mu \mathrm{m}$ [146], already available for production [147], will increase the dynamic range [149, 150] improving the resolution for heavy nuclei charge measurement (opening the possibility to identify trans-Iron elements) and may feature higher resilience towards radiation damage $[151,152]$. The interest in SiPMs for space payloads is evident from the variegate amount of past, present and future R\&D activities [153-155]. Results from ground tests of SiPM readout of scintillator bars with similar dimensions to those foreseen for ALADInO already show promising time resolutions much better than 100 ps $[156,157]$;

Timing layers Low Gain Avalanche Diode (LGAD) Si sensors [158], which feature thinner layers (i.e., less material budget) with the same, or even higher, signal/noise electronic level, could be considered to replace standard micro-strip Si sensors. This technology may provide timing capabilities with performances similar to those of the ToF (i.e. $<100 \mathrm{ps)}$ to the tracking layers, providing the apparatus with a second time-offlight detector to improve the overall performances (e.g. isotopical distinction) and give further redundancy to cross-calibrate the detectors. The timing capability will also allow to identify energy deposits in the tracking layers due to back-scattering particles from the calorimeter, further improving the overall identification capabilities of the apparatus [159];

Pixel technology Monolithic Active Pixel Sensors (MAPS) [160] represent a possible replacement of micro-strip Si detectors. MAPS do not suffer multi-hit degeneracy and are thinner than micro-strips, limiting multiple scattering effects [161]. They feature inpixel amplification, with significant gain in terms of signal-to-noise ratio and data throughput optimization. MAPS pixel dimensions can ensure single-hit resolution down to $1 \mu \mathrm{m}$ [162]. Large-scale fabrication processes and infrastructures shared with image sensors for mobile phone cameras, reduce the cost to 10 times lower than current micro-strip detectors. Similar application of MAPS technology (ALPIDE [163]) for space application is also considered, for example, for the HEPD-02 tracker to be launched on board the CSES-02 satellite in 2021 [164] and for the Timepix series hybrid pixel detectors [165] for dosimetry measurements and in space [166] and space weather analyses [167]. Future developments of MAPS focus on the reduction of power consumption (halving every 3 years since 2002 [168, 169]), and techniques for large area production (hundreds of $\mathrm{cm}^{2}$ ). It is worth recalling that MAPS feature distributed on-chip intelligence, allowing event-driven readout solutions, further reducing power consumption and simplifying the operation [170].

Capability for $\gamma$-rays the spectrometer can be also used as an excellent tracker-converter for $\gamma$-rays. The $\mathrm{e}^{+} / \mathrm{e}^{-}$pairs produced by conversion of $\gamma$-rays with the silicon tracker material are traced and their momentum measured by the spectrometer. The low material budget guarantees a PSF better than 0.5 deg down to the lowest energies $(\sim 100 \mathrm{MeV})$, consistent with what is expected for similar proposed detectors [171]. Despite of the limited conversion probability due to the low amount of material, the large spectrometer geometrical factor will result in a $\sim 0.5 \mathrm{~m}^{2} \mathrm{sr}$ effective acceptance, effectively enabling for competitive measurements in the low energy $\gamma$-ray field [172].

Orbital requirements Access to space is necessary to avoid antimatter background from CR collisions with the Earth's atmosphere. Geomagnetic flux modulation, 
back-scattered albedo particles, and geomagnetically trapped particles represent known limiting factors for low-Earth orbiting experiments that affect acquisition capabilities (via radiation damage) and CR flux measurement (via Galactic CR flux suppression at $\mathrm{p} /|\mathrm{Z}|<\sim 30 \mathrm{GV})$. The latter is critical for the observational goals related to low energy anti-deuteron, anti-helium measurements, and heliospheric studies. All these issues can be resolved by a high-Earth orbit or L2 orbit. Moreover, these types of orbit are most appropriate for a proper thermal environment required by the scientific payload. It is important to note that no active attitude control is required for the mission, provided that appropriate sky coverage is guaranteed.

Weight and power budget Considering the technology currently available, about 2 tons of the mass budget are allocated to the magnetic system, including cryogenics and insulation. The next heavy part is the LYSO crystal of the calorimeter, weighting 2.3 tons including $300 \mathrm{~kg}$ of mechanical structure. The Tracker and ToF systems are estimated to weigh about 1.5 ton and about $500 \mathrm{~kg}$ are allocated for the power, readout and control electronics of all the subsystems. The current total mass estimate is thus about 6.4 tons for the experimental apparatus. This mass estimation does not include the improvements expected during the next years by ongoing R\&D on most of the subsystems.

The ALADInO detector, in spite of its high acceptance has a limited number of readout channels, thanks to its innovative design, but still is quite demanding in term of power for a space application. The most consuming detector subsystem is the Tracker, with $\sim 2000,000$ channels and a total power consumption of about $5 \mathrm{~kW}$. The Calorimeter is read-out through 31,000 electronics channels, with an estimated power consumption of about $200 \mathrm{~W}$. The ToF, with about 2000 channels of high-speed electronics has a power consumption of about $400 \mathrm{~W}$. The cryogenic system will consume about $1 \mathrm{~kW}$ to keep the magnets coil at the nominal operating temperature. The total power budget is thus estimated to be $\sim 12 \mathrm{~kW}$. The power consumption estimation, however, does not include the improvements expected during the next years by ongoing R\&D on most of the subsystems.

Pathfinder strategy The physics goals together with the technology development needs, suggest a staging and technology demonstrator strategy which would likely better suit ESA's long term science program. The pathfinder version of ALADInO would be based on a superconducting magnet with a reduced magnetic field (about 10 times) but with the same collecting area: from the science point of view this demonstrator would be able to address the important theme of nuclear antimatter up to 100 GV of rigidity, providing a MDR of $1 \mathrm{TV}$. The magnet weight would be reduced to about $400 \mathrm{~kg}$ : assuming a $20 \%$ weight reduction due to R\&D optimization for the Tracker and the ToF subsystems, the pathfinder weight would be about 2.0 tons, less than a third of the weight of the full version, still providing first class science.

Operational requirements The experiment must be operational $24 \mathrm{~h}$ a day, 7 days a week, and 365 days a year without human interventions or on-orbit maintenance activities. It must provide a continuous stream of data at a rate of the order of $\sim 50$ Mbps. This requires the development of sophisticated onboard data reduction and compression techniques and related hardware. 


\section{Concluding remarks}

Astroparticle physics is a rich field of study that addresses big science questions at the intersection of astrophysics and particle physics. Observationally, it involves making astrophysical measurements using multiple cosmic messengers such as charged particles, $\gamma$-rays, neutrinos, and gravitational waves. These messengers can be used to test fundamental physics under extreme conditions, in otherwise inaccessible environments, or at energies beyond the reach of laboratory experiments.

In this context, the advent of the era of high precision cosmic rays provided by the use of space spectrometers like AMS-02 opens a window of sensitivity which can uniquely address issues at the core of our understanding of the Universe. The proposed physics program lies in the study of cosmological, particle physics, and high-energy astrophysical phenomena focusing on the detection of charged antiparticles and particles in space. The ALADInO program addresses two of the main unsolved problems of modern astrophysics - the missing antimatter and the dark matter puzzles - but it also encompasses a wide range of exciting problems: the propagation of cosmic rays in galactic turbulence, their transport throughout the heliospheric plasma, the physics mechanism of particle acceleration in astrophysical shockwaves, and the technological challenges associated with the detection of antiparticles in space. One hundred years after the discovery of Cosmic Rays by V. Hess, ALADInO science will fully exploit the fully potential to address some of the hottest astrophysical and cosmological questions of the XXI ${ }^{\text {st }}$ century.

Code availability NA

Authors' contributions NA

Funding Open access funding provided by Universit $\tilde{A}$ degli Studi di Trento within the CRUI-CARE Agreement.

Data availability NA

\section{Declarations}

\section{Conflict of interest NA}

Open Access This article is licensed under a Creative Commons Attribution 4.0 International License, which permits use, sharing, adaptation, distribution and reproduction in any medium or format, as long as you give appropriate credit to the original author(s) and the source, provide a link to the Creative Commons licence, and indicate if changes were made. The images or other third party material in this article are included in the article's Creative Commons licence, unless indicated otherwise in a credit line to the material. If material is not included in the article's Creative Commons licence and your intended use is not permitted by statutory regulation or exceeds the permitted use, you will need to obtain permission directly from the copyright holder. To view a copy of this licence, visit http://creativecommons.org/licenses/by/4.0/.

\section{References}

1. Battiston, R.: La. Rivista del Nuovo Cimento. 43(7), 319-384 (2020)

2. Blinnikov, S.I., et al.: Phys. Rev. D. 92, 023516 (2015)

3. Dolgov, A.D.: Gravitation, Astrophysics. Cosmol. 168-175 (2016) 
4. Poulin, V., et al.: Phys. Rev. D. 99, 023016 (2019)

5. Cirelli, M.: Proc. 34th ICRC, PoS (ICRC2015)014 (2015)

6. Salati, P.: Proc. 17th Lomonosov conference, Moscow [arXiv:1605.01218] (2015)

7. Cirelli, M., et al.: JCAP. 1510, 026 (2015)

8. Boschini, M. et al.: Astrophysical J. 840(2), 115 (2017)

9. Boudad, M. et al.: arXiv:1906.07119 (2019)

10. Blasi, P.: Braz. J. Phys. 44, 426; [arXiv:1312.1590, see also arXiv:1412.8430] (2014)

11. Grenier, I.A., et al.: Ann. Rev. A\&A. 53, 199 (2015)

12. Potgieter, M.: Living rev. Sol. Phys. 10, 3 (2013)

13. Bisschoff, D. et al.: Astrophys. J. 878(1), 59 (2019)

14. Ding, Y.-C.: JCAP 06(2019), 004 (2019)

15. Abe, K. et al., (BESS Collaboration): Phys. Lett. B 670(2), (2008)

16. Alcaraz, J. (AMS-01 Collaboration) et al.: Phys. Lett. B 461, 387 and references therein (1999)

17. Adriani, O., (PAMELA Collaboration) et al.: Phys. Rep. 544, 323-370 (2014)

18. Cohen, A.G., et al.: Astrophys. J. 495, 539 (1998)

19. Sakharov, A.D.: JTEP Lett. 5, 24 (1967)

20. Bambi, C., Dolgov, A.D.: Nucl. Phys. 784, 132 (2007)

21. Kounine, A., PoS (ICRC2017) 1093 (2017)

22. Ade, N., et al.: A\&A. 594, A13 (2016)

23. Akrami, Y., et al.: arXiv. 1807.06211 (2018)

24. Ostdiek, B.: Phys. Rev. D. 92, 055008 (2015)

25. Boveia, A., et al.: Ann. Nucl. Part. Sci. 429-459, 68 (2018)

26. Cirelli, M., et al.: JHEP. 1408, 009 (2014)

27. Hisano, J., et al.: JHEP. 1506, 097 (2015)

28. Masi, N., Ballardini, M., Int. J. Mod. Phys. D 0, 1750041 (2016)

29. Ajello, M., FERMI Collaboration, et al.: Astrophys. J. 819, 44 (2016)

30. Albert, A., FERMI Collaboration, et al.: Astrophys. J. 834, 110 (2017)

31. Cholis, I., Hooper, D., Linden, T.: Phys. Rev. D. 99, 103026 (2019)

32. Conrad, J., Reimer, O.: Nat. Phys. 13, 224-231 (2017)

33. Turner, M.S., Wilczek, F.: Phys. Rev. D42, 1001-1007 (1990)

34. Ellis, J.R.: AIP Conf. Proc. 516(2000), 21 (2000)

35. Cheng, H.-C., et al.: Phys. Rev. Lett. 89, 211301 (2002)

36. Cirelli, M., et al.: Nucl. Phys.B. 813, 1 (2009)

37. Kane, G., et al.: Phys. Lett. B681, 151-160 (2009)

38. Kopp, J.: Phys. Rev. D88, 076013 (2013)

39. Belotsky, K., et al.: Adv. High Energy Phys. 2014, 214258 (2014)

40. Chen, C.-H., et al.: Phys. Lett. B747, 495-499 (2015)

41. Bhattacharyya, S., et al.: JCAP. 1708(08), 012 (2017)

42. Cheng, H.-C., et al.: JCAP. 1703, 041 (2017)

43. Bai, Y., et al.: Phys. Rev. D97, 115012 (2018)

44. Feng, J., Zhang, H.-H.: Astrophys. J. 858(2), 116 (2018)

45. Profumo, S., et al.: Phys. Rev. D97, 123008 (2018)

46. Fujita, Y., et al.: Phys. Rev. D80, 063003 (2009)

47. Hooper, D., et al.: J. Cosmol. Astropart. Phys. 1, 025 (2009)

48. Serpico, P.: Proc. 34th Int. cosmic ray Conf., PoS (ICRC2015) 009 (2015)

49. Linden, T., Profumo, S.: Astrophys. J. 772, 18 (2013)

50. Mertsch, P., Sarkar, S.: Phys. Rev. D90, 061301 (2014)

51. Tomassetti, N.: ApJ 815, L1 (2015) [arXiv: 1511.04460]

52. Tomassetti, N., Donato, F.: ApJ Lett. 803, L15 (2015) [arXiv:1502.06150]

53. Hooper, D., et al.: Phys. Rev. D96, 103013 (2017)

54. Liu, W., et al.: Phys. Rev. D96, 023006 (2017)

55. Kachelrieß, M., et al.: Phys. Rev. D97, 063011 (2018)

56. Bykov, A.M., et al.: Astrophys.J. 876(1), L8 (2019)

57. Fornieri, O. et al.: arXiv:1907.03696 (2019)

58. Profumo, S., et al.: arXiv:1903.07638 (2019)

59. Cowsik, R.R., Burch, B.: Phys. Rev. D82, 023009 (2010)

60. Katz, B., et al.: Mon. Not. R. Astron. Soc. 405, 1458 (2010)

61. Lipari, P.: Phys. Rev. D95, 063009 (2017)

62. Blum, K., et al.: Phys. Rev. Lett. 111, 211101 (2013) 
63. Kruskal, M., et al.: Astrophys. J. 818, 70 (2016)

64. Diesing, R., Caprioli, D.: arXiv:1905.07414 (2019)

65. Lipari, P.: Phys. Rev. D99(4), 043005 (2019)

66. Gabici, S., et al.: arXiv:1903.11584 (2019)

67. Yoon, Y.S., CREAM-III Collaboration, et al.: ApJ. 839, 5 (2017)

68. Adriani, O.: CALET Collaboration, et al.: Phys. Rev. Lett. 122, 181102 (2019)

69. Yan, Q.: Contribution to 36th international cosmic ray conference (ICRC2019) (2019)

70. Tomassetti, N.: Phys. Rev. D. 92, 063001 (2015) [arXiv:1509.05774]

71. Kulikov, G.V., Khristiansen, G.B.: J. Exp. Theor. Phys. 35, 635 (1958)

72. Bluemer, J., et al.: Prog. Part. Nucl. Phys. 63, 293-338 (2009)

73. D'Enterria, D., et al.: Astrop. Phys. 35, 98 (2011)

74. Hoerandel, J.R.: Astrop. Phys. 21, 241 and references therein (2004)

75. Gaggero, D., et al.: Astrophys. J. 815(2), L25 (2015)

76. Seo, E.S.: Astrop. Phys. 39-40, 76 (2012)

77. Abe, K.: (BESS Collaboration), et al.: Phys. Rev. Lett. 108, 051102 (2012)

78. Adriani, O.: (PAMELA Collaboration), et al.: Nature. 458, 607 (2009)

79. Adriani, O., (PAMELA Collaboration), et al.: Phys. Rev. Lett. 111, 081102 (2013)

80. Aguilar, M., (AMS-02 Collaboration), et al.: Phys. Rev. Lett. 110, 141102 (2013)

81. Aguilar, M., (AMS-02 Collaboration), et al.: Phys. Rev. Lett. 113, 221102 (2014)

82. Aguilar, M.: (AMS-02 Collaboration), et al.: Phys. Rev. Lett. 122(4), 041102 (2019)

83. Aguilar, M., (AMS-02 Collaboration), et al.: Phys. Rev. Lett. 122(10), 101101 (2019)

84. Marocchesi, P.S., et al.: J. Phys. Conf. Ser. 718, 052023 (2016)

85. Chang, J., et al.: Astropart. Phys. 95, 6-24 (2017)

86. Zhang, S. et al.: PoS (ICRC2017) 1077 (2017)

87. M. Aguilar (AMS-02 Collaboration) et al.: Phys. Rev. Lett. 117, 091103 (2016)

88. Jin, H. et al.: arXiv:1701.02213; H. Jin et al., 2015, Phys. Rev. D 92, no. 5, 055027 (2017)

89. Cui, M.Y., et al.: Phys. Rev. Lett. 118, 191101 (2017)

90. Cuoco, A., et al.: Phys. Rev. Lett. 118, 191102 (2017)

91. Cuoco, A., et al.: Phys. Rev. D. 99, 103014 (2019)

92. Cowsik, R.R., Madziwa-Nussinov, T.: Astrophys. J. 827, 119 (2016)

93. Tomassetti, N., Oliva, A.: ApJ Lett. 844 [arXiv:1707.06915] (2017)

94. Lu, B.Q., Zong, H.S.: Phys. Rev. D. 93, 103517 (2016)

95. Aramaki, T., et al.: Phys. Report. 618(7), 1-37 (2016)

96. Cirelli, M., et al.: JHEP. 1410, 033 (2014)

97. Carlson, E., et al.: Phys. Rev. D. 89, 076005 (2014)

98. Coogan, A., Profumo, S.: Phys. Rev. D. 96, 083020 (2017)

99. Korsmeier, M., et al.: Phys. Rev. D. 97(10), 103011 (2018)

100. Sakai, K., et al, PoS(ICRC2019) (2019)

101. Aharonian, F., (HESS Collaboration), et al.: Astron. Astrophys. 508, 561 (2009)

102. Staszak, D., (VERITAS collaboration) et al.: PoS(ICRC2015) 411 (2015)

103. Tridon, D., et al.: (MAGIC Collaboration) Proceedings of the 32nd ICRC (2011)

104. Ambrosi, G., (DAMPE Collaboration), et al.: Nat. 552, 63-66 (2017)

105. Adriani, O., CALET Collaboration, et al.: Phys. Rev. Lett. 119, 181101 (2017)

106. Adriani, O., CALET Collaboration, et al.: Phys. Rev. Lett. 120, 261102 (2018)

107. Abdollahi, S., Fermi Collaboration, et al.: Phys. Rev. D. 95, 082007 (2017)

108. Athron, P., et al.: JHEP. 1802, 121 (2018)

109. Fan, Y.: Phys. Lett. B781, 83-87 (2018)

110. Jin, H., et al.: Phys. Rev. D98(12), 123008 (2018)

111. Liu, X., Liu, Z.: Phys. Rev. D98(3), 035025 (2018)

112. López-Coto, R., et al.: Phys. Rev. Lett. 121, 251106 (2018)

113. Niu, J.: Eur. Phys. J. C79(2), 125 (2019)

114. Mertsch, P.: JCAP. 1811(11), 045 (2018)

115. Recchia, S., et al.: Phys. Rev. D. 99(10), 103022 (2019)

116. Kounine, A. et al.: Nucl., Instrum. Meth. A. 869, 100-117 (2017)

117. Acharya, B.S. et al.: (CTA Consortium), ISBN: 978-981-3270-08-4. arXiv:1709.07997 (2019)

118. Serpico, P.D.: Astropart. Phys. 39-40, 2-11 (2012)

119. Parsons, R.D., et al.: Astropart. Phys. 84, 23-28 (2016)

120. Adriani, O., PAMELA Collaboration, et al.: Science. 332, 69 (2011)

121. Aguilar, M., AMS-02 Collaboration, et al.: Phys. Rev. Lett. 114, 171103 (2015) 
122. Aguilar, M., AMS-02 Collaboration, et al.: Phys. Rev. Lett. 115, 211101 (2015)

123. Aguilar, M., AMS-02 Collaboration, et al.: Phys. Rev. Lett. 119, 251101 (2017)

124. Kachelrieß, M., Semikoz, D. V.: arXiv: 1904.08160 (2019)

125. Alvisi, D., et al.: Nucl. Instrum. Meth. A. 437, 212-221 (1999)

126. Alcaraz, J., et al.: Nucl. Instrum. Meth. A. 593, 376-398 (2008)

127. Osteria, G. et al.: Nucl. Instrum. Meth. A. 161-163 (2004)

128. Straulino, S., et al.: Nucl. Instrum. Meth. A. 556, 100-114 (2006)

129. Atwood, W., FERMI Collaboration, et al.: ApJ. 697(2), 1071-1102 (2009)

130. Bigongiari, G., CALOCUBE collaboration, et al.: Univ. 5(3), 72 (2019)

131. Bigongiari, G. CALOCUBE collaboration: Proc.18th Lomonosov Conf. on Elem. Part. Phys. 300-304 (2019)

132. Majkic, G., et al.: Nature. Sci. Rep. 8, 6982 (2018)

133. Musenich, R. et al.: IEEE Trans. Appl. Supercond. 24 (3) (2014)

134. Kovác, P., et al.: Supercond. Sci. Technol. 31, 095006 (2018)

135. Zhang, X., et al.: J. Supercond. Nov. Magn. 31(11), 3465-3474 (2018)

136. Musenich, R. et al.: IEEE Trans. Appl. Supercond. 26 (4) (2016)

137. Bruce, R., Baudouy, B.: Phys. Procedia. 67, 2015 (2015)

138. Juster, F.P., Berriaud, C.: SR2S report SR2S-WP31-D31.4 (2015)

139. Dam, M., et al.: Supercond. Sci. Technol. 33, 044012 (2020)

140. L. Rossi private communication

141. Moery, J.: Proc. SPIE, space telescopes and instrumentation I: optical, infrared, and millimeter, 6265 , 62653B (2006)

142. Bruce, R., et al.: Cryogenics. 93, 66-74 (2018)

143. Azzarello, P., et al.: Nucl. Instrum. Meth. A. 831, 378-384 (2016)

144. Ambrosi, G., et al.: Nucl. Instrum. Meth. A. 869, 29-37 (2017)

145. Dorosz, P., et al.: IEEE. Trans. Nucl. Sci. 65(4), 1070-1078 (2018)

146. Piemonte, C., Gola, A.: Nucl. Instrum. Meth. A. 926, 2-15 (2018)

147. Gola, A., et al.: Sens. 19(2), 308 (2019)

148. Acerbi, F. et al.: Proc. SPIE, 10212, Advanced Photon Counting Techniques XI, 102120I (2017)

149. Acerbi, F., et al.: IEEE J. Quantum Electron. 54(2), 4700107 (2018)

150. Bretz, T., et al.: J. Instrum. 11, P03009 (2016)

151. Link, J. et al.: PoS (ICRC2017) 0235 (2017)

152. Garutti, E., Musienko, Y.: Nucl. Instrum. Meth. A. 926, 69-84 (2019)

153. Kim, M., et al.: Nucl. Instrum. Meth. A. 703, 177-182 (2013)

154. Arkhangelskiy, A., et al.: Nucl. Instrum. Meth. A. 74, 390-393 (2015)

155. Perrina, C.: EPJ Web Conf. 209, 01040 (2019)

156. Böhm, M., et al.: Nucl. Instrum. Meth. A. 912, 323-325 (2018)

157. Betancourt, C., et al.: J. Instrum. 12, P11023 (2017)

158. Cartiglia, N. et al.: IFD2015, 026, (http://pos.sissa.it/266/026/pdf) (2015)

159. Duranti, M.: Contribution to TREDI2019, (http://tiny.cc/TREDI2019/DTP.pdf) (2019)

160. Turchetta, R., et al.: Nucl.Instrum.Meth. A458, 677-689 (2001)

161. Mager, M., et al.: Nucl. Instrum. Meth. A824, 434438 (2016)

162. Arai, Y.: Jpn. J. Appl. 57, 1002A1 (2018)

163. Iuppa, R.: PoS(ICRC2019) 083 (2019)

164. Ballabriga, R., et al.: Nucl. Instrum. Meth. A878, 10-23 (2018)

165. Turecek, D., et al.: J. Instrum. 6, C12037 (2011)

166. Gohl, S., et al.: J. Instrum. 11, C11023 (2016)

167. Baudot, J. et al.: IEEE Nuclear Science Symposium conference, 5402399 (2009)

168. Ricci, E., et al.: Il Nuovo Cimento. 42 C, 209 (2019)

169. Wu X., et al.: PANGU collaboration (sci.esa.int/science-e/www/object/doc.cfm?fobjectid=54974) (2014)

170. Tavani, M., e-ASTROGAM collaboration, et al.: SPIE Conf. Ser. 4851, 1151-1162 (2003)

171. Aguilar, M., AMS-02 Collaboration, et al.: Phys. Rev. Lett. 120, 021101 (2018)

172. Mori, N., et al.: Nucl. Instrum. Meth. A. 732, 311-315 (2013)

173. Agostinelli, S., et al.: Nucl. Instrum. Meth. A. 506, 250 (2003)

174. Allison, J., et al.: IEEE trans. Nucl. Sci. 53, 270 (2006) 
Publisher's Note Springer Nature remains neutral with regard to jurisdictional claims in published maps and institutional affiliations.

\section{Affiliations}

R. Battiston ${ }^{1,2} \cdot$ B. Bertucci ${ }^{3,4} \cdot$ O. Adriani ${ }^{5,6} \cdot$ G. Ambrosi $^{4} \cdot$ B. Baudouy ${ }^{7} \cdot \mathrm{P}$. Blasi $^{8} \cdot$ M. Boezio ${ }^{9}$ - D. Campana ${ }^{10}$ - L. Derome ${ }^{11} \cdot$ I. De Mitri ${ }^{8} \cdot$ V. Di Felice ${ }^{12} \cdot$ F. Donato $^{13} \cdot$ M. Duranti ${ }^{3} \cdot$ V. Formato ${ }^{12} \cdot$ D. Grasso ${ }^{14} \cdot$ I. Gebauer $^{15} \cdot$ R. Iuppa ${ }^{1,2}$. N. Masi ${ }^{17} \cdot$ D. Maurin ${ }^{11} \cdot$ M. N. Mazziotta ${ }^{17} \cdot$ R. Musenich $^{18} \cdot$ F. Nozzoli ${ }^{2} \cdot$ P. Papini $^{16} \cdot$ P. Picozza ${ }^{19,12} \cdot$ M. Pearce ${ }^{20} \cdot$ S. Pospíšil ${ }^{21} \cdot$ L. Rossi ${ }^{22} \cdot$ N. Tomassetti $^{3,4} \cdot$ V. Vagelli ${ }^{23} \cdot \mathrm{X} . \mathrm{Wu}^{24}$

\section{R. Battiston}

roberto.battiston@unitn.it

University of Trento, Trento, Italy

2 INFN-TIFPA, Trento, Italy

3 University of Perugia, Perugia, Italy

4 INFN Section of Perugia, Perugia, Italy

5 University of Florence, Florence, Italy

6 INFN Section of Florence, Florence, Italy

7 CEA Irfu/SACM, Saclay, France

8 Gran Sasso Science Institute \& INFN National Laboratory of Gran Sasso, L'Aquila, Italy

9 INFN Section of Trieste, Trieste, Italy

10 INFN Section of Neaples, Neaples, Italy

11 University Grenoble Alpes and IN2P3 LSPC, France, Italy

12 INFN Section of Rome Tor Vergata, Rome, Italy

13 University and INFN Section of Turin, Turin, Italy

14 INFN Section of Pisa, Pisa, Italy

15 KIT, Karlsruher Institut für Technologie, Karlsruhe, Germany

16 University and INFN Section of Bologna, Bologna, Italy

17 INFN Section of Bari, Bari, Italy

18 INFN Section of Genoa, Genoa, Italy

19 University of Roma Tor Vergata, Rome, Italy

20 KTH Royal Institute of Technology, Stockholm, Sweden

21 CTU, Czech Technical University, Prague, Czechia

22 CERN, Meyrin, Switzerland

ASI, Italian Space Agency, Rome, Italy

24

University of Geneva, Geneva, Switzerland 